\title{
Efficient Modeling of Microwave Integrated-Circuit Geometries via a Dynamically Adaptive Mesh Refinement-FDTD Technique
}

\author{
Yaxun Liu, Member, IEEE, and Costas D. Sarris, Member, IEEE
}

\begin{abstract}
The finite-difference time-domain (FDTD) method is combined with an adaptive mesh refinement (AMR) technique, to achieve a fast, time-domain solver for Maxwell's equations (AMRFDTD), based on a three-dimensional moving/rotating Cartesian mesh. This combination allows the proposed solver to adapt to the problem at hand, optimally distributing computational resources in a given domain as needed, by recursively refining a coarse grid in regions of large over time gradient of electromagnetic field energy. Several applications of the method to the analysis of microwave circuit geometries demonstrate its salient features and its outstanding efficiency as a microwave computer-aided design tool.
\end{abstract}

Index Terms-Adaptive mesh refinement (AMR), finite difference time domain (FDTD), microwave integrated circuits.

\section{INTRODUCTION}

$\mathbf{T}$ HE finite-difference time-domain (FDTD) technique [1] has been extensively employed in the modeling of microwave integrated circuits [2]. It is especially suitable for wideband applications since it allows for the characterization of a given structure in a broad frequency range, through a single simulation. However, the FDTD stability and dispersion properties impose severe limitations on the choice of the cell size and the time step of the method, rendering its application to complex structures computationally expensive.

The challenge of accelerating FDTD simulations for practical geometries has been addressed in the past with a variety of static subgridding techniques [3]-[6]. According to those, local mesh refinement is pursued in a priori defined regions of a computational domain, as dictated by physical considerations. For example, the presence of metallic edges or high-dielectric permittivity inclusions would call for a locally dense mesh, embedded in a coarser global one. The use of local mesh refinement typically results in significant computational savings compared to the conventional FDTD method, despite the fact that its implementation is associated with additional interpolation and extrapolation operations in both space and time.

However, this approach ignores the dynamic nature of timedomain field simulations. In fact, techniques such as FDTD and transmission-line matrix (TLM) essentially register the history of a broad-band pulse propagating in a device-under-test (DUT),

Manuscript received June 21, 2005; revised September 16, 2005. This work was supported by the Natural Sciences and Engineering Research Council of Canada under a Discovery Grant.

The authors are with the Edward S. Rogers Sr. Department of Electrical and Computer Engineering, University of Toronto, Toronto, ON, Canada M5S 3G4 (e-mail: cds@waves.utoronto.ca).

Digital Object Identifier 10.1109/TMTT.2005.862660 along with its multiple reflections from parts of the latter. Hence, a sharp edge of a microstrip structure is not continuously illuminated by the pulse excitation; on the contrary, it is so for a (potentially small) fraction of the total simulation time, during which a local mesh refinement around it is needed. Therefore, static mesh refinement, which is widely employed in frequencydomain simulations and has been incorporated in commercial finite-element tools, is only a suboptimal solution to the mesh refinement problem in the framework of time-domain analysis.

More recently, a moving-window finite-difference time-domain (MW-FDTD) method was proposed for the tracking of the forward propagating wave in the two-dimensional (2-D) terrain environment of a wireless channel [7]. The single moving window used by the method was characterized by fixed size and velocity and, therefore, it could not track reflections (which were absorbed by terminating boundaries of the window). As a result, the MW-FDTD is is not well suited for microwave circuit simulations, where the modeling of phenomena as common as signal reflection and branching would require multiple and potentially rotating windows.

In the context of computational fluid dynamics, the technique of adaptive mesh refinement (AMR) was introduced in [8], for the solution of hyperbolic partial differential equations. The application of AMR is based on the use of a hierarchical mesh, which is recursively developed through the refinement of a coarse root mesh, which covers the entire computational domain. The regions of the computational domain that need further mesh refinement are detected via error estimates or indicators such as gradients of the quantity for which to be solved. There may also be dense mesh regions, where the use of a dense mesh is not necessary after a certain time step. These can then be coarsened, again in a recursive manner. Dense and coarse mesh regions are organized via a clustering algorithm that is accompanied by regular checks (every certain time steps) of the error estimates, which guide the process of migration of a cell from one level of resolution to another. This procedure can be associated with the algorithm of [9] and [10], which used wavelet field expansions in order to track the spatio-temporal evolution of shock-wave and nonlinear optical pulse propagation problems, respectively. However, the generalization of such wavelet-based algorithms to three dimensions presents a significant added complexity, while the implicit relation between the actual field values and the wavelet expansion coefficients renders the application of boundary conditions, which are essential for the connection of nested meshes of different resolutions, computationally burdensome. 
In this paper, the conventional FDTD is combined with the AMR method, in order to formulate an efficient AMR-FDTD technique of superior performance, extending earlier work by the authors [11], [12]. Instead of using fixed subgrids, this method uses subgrids that are adaptively defined, according to the evolution of field distributions in space and time. As an example, when a Gaussian pulse propagating along a microstrip line is simulated, the AMR scheme successfully tracks the movement of the pulse, thereby refining only the region that surrounds the propagating pulse. In this case, the AMR accuracy is comparable to that of a uniformly (throughout the entire computational domain) dense mesh FDTD.

The paper is organized as follows. The general structure of the AMR-FDTD algorithm is presented in Section II, while Section III refers to the update procedure of the tree of meshes that the algorithm uses in order to adaptively track the field evolution. Section IV outlines how cells that need further mesh refinement or cells that can be removed from the mesh are detected, clustered, and managed by the AMR-FDTD scheme. Finally, Section V presents an extensive set of time- and frequency-domain numerical results for realistic applications, which illustrate the advantages of the algorithm. Based on these results, accuracy and stability aspects of the technique are presented.

\section{AMR-FDTD: OVERVIEW OF THE ALGORITHM}

In general, a Cartesian FDTD mesh occupies a rectangular region

$$
A=\left\{(x, y, z), x_{a_{1}} \leq x \leq x_{a_{2}}, y_{a_{1}} \leq y \leq y_{a_{2}}, z_{a_{1}} \leq z \leq z_{a_{2}}\right\}
$$

that is terminated by closed or absorbing boundaries. Inhomogeneous material properties can be readily assumed by letting the dielectric permittivity and magnetic permeability of the structure be generic functions of the space variables $\epsilon=\epsilon(x, y, z), \mu=\mu(x, y, z)$, respectively. Let us consider the subdivision of the domain $A$ in $N_{x} \times N_{y} \times N_{z}$-Yee cells, indexed by a triplet $(i, j, k)$. Each cell occupies a volume $\Delta x \times \Delta y \times \Delta z$, where $\Delta x, \Delta y, \Delta z$ denote the cell sizes in the $x, y$, and $z$ directions. The mesh of these $N_{x} \times N_{y} \times N_{z}$ cells is the coarse grid of region $A$ that the algorithm under development will selectively and locally refine.

Let us now assume that there are cells within the domain $A$ whose refinement is necessary, according to certain accuracy criteria (the discussion of the latter is deferred to Section IV). These cells are first clustered together and then covered by rectangular subregions $B_{n}, n=1,2, \ldots, N$ which belong to $A$. Throughout the algorithmic development of the AMR-FDTD, it will be ensured that these subregions can share planar boundaries, yet they cannot overlap. This is important in order to preserve the possibility of further refinement of these subregions independently from each other, as required by the evolution of the field solution. Each rectangular region $B_{n}$ is subdivided in Yee cells of dimensions: $\Delta x / 2, \Delta y / 2, \Delta z / 2$. Hence, a refinement factor of 2 is used in every direction, reducing the Yee cell volume of the initial mesh by a factor of 8 .

To summarize, a coarse mesh has been defined in the rectangular region $A$, enclosing finer meshes in rectangular subregions $B_{n}$ of the latter. The mesh of region $A$, henceforth re-

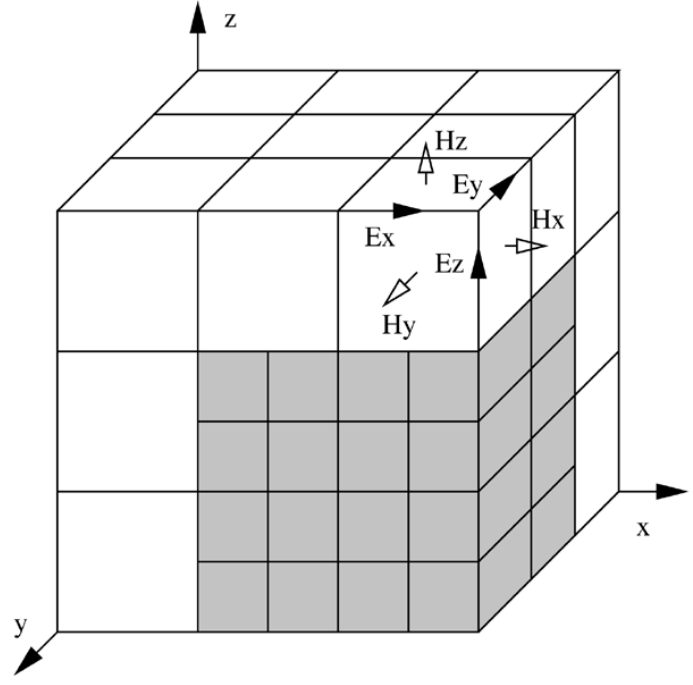

Fig. 1. Sampling points of electric and magnetic field components in a Yee cell. Solid arrows are for electric field, whereas hollow arrows are for the magnetic field. The shaded areas represent refined Yee cells.

ferred to as mesh $A$, will be called the root mesh or level 1 mesh. The meshes of regions $B_{n}$, or meshes $B_{n}$, will be called child meshes of $A$ or level 2 meshes. Recursively, each $B_{n}$ can be further refined to have its own child meshes, again refining the cell sizes involved by a factor of 2 . Therefore, all of the meshes that are generated in this manner can be assembled in a tree structure, since its hierarchy has a one-to-one correspondence with the proposed order of AMR-FDTD grids.

According to the convention of the Yee cell in FDTD (Fig. 1), electric field components are sampled at the center of the edges of each cell, while magnetic field components are sampled at face centers. The sampling points of a parent mesh may coincide or not with the sampling points of its child mesh, depending on whether the refinement factor is odd or even, respectively. In this study, the refinement factor is 2 (or powers of 2, with respect to the root mesh), and, hence, the grid points of child and parent meshes do not coincide. An alternative case, where the choice of a refinement factor of three renders the parent mesh sampling points also child mesh sampling points, can be found in [6].

The main difference between standard subgridded FDTD methods and AMR-FDTD is the dynamic mesh generation which is pursued in the latter, every $N_{\mathrm{AMR}}$ time steps. In order to define the time step of AMR-FDTD, the following observations need to be made. For a level $M$ mesh, Yee cell dimensions are: $\Delta x / 2^{M-1}, \Delta y / 2^{M-1}, \Delta z / 2^{M-1}$, where $\Delta x, \Delta y, \Delta z$ are the root mesh Yee cell dimensions. Furthermore, the Courant number is fixed to a constant value $s$ in all meshes. This implies that the root mesh time step $\Delta t$ (from now on referred to as AMR-FDTD time step) is given as

$$
\Delta t=s \frac{1}{u_{p}^{\max }} \frac{1}{\sqrt{\frac{1}{\Delta x^{2}}+\frac{1}{\Delta y^{2}}+\frac{1}{\Delta z^{2}}}}
$$

where $u_{p}^{\max }$ is the maximum phase velocity in the computational domain. Applying (1) for a mesh of level $M$, keeping $s$ fixed, yields a time step $\Delta t_{M}$ for this mesh equal to

$$
\Delta t_{M}=\Delta t / 2^{M-1} \text {. }
$$




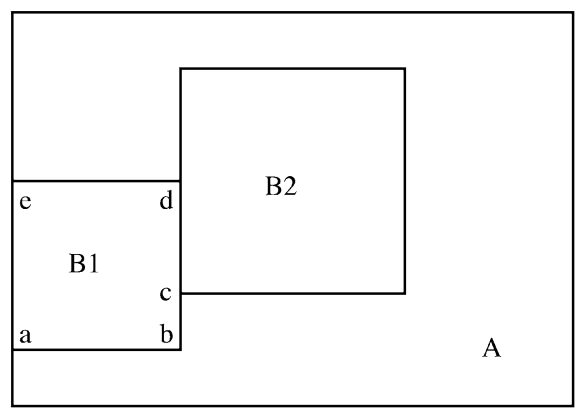

Fig. 2. Types of boundaries of a child mesh.

For example, level 2 meshes are updated twice as many times as the root mesh. Thus, another shortcoming of the conventional FDTD is addressed; the minimum time step of the algorithm is only used for the update of regions of large field variations, as opposed to the whole domain, which is a salient feature that is also part of the fixed subgridding algorithms of [3] and [6].

The loop of the AMR-FDTD operations is as follows.

Step 1) Check the number of time steps executed. If it is an integer multiple of $N_{\mathrm{AMR}}$, perform adaptive mesh refinement to create a new mesh tree, and carry the field values from the old mesh tree to the new mesh tree.

Step 2) Update fields of the root mesh.

Step 3) Copy fields from the root mesh to the boundary of the child meshes. Update fields of the child meshes $2^{M-1}$ times, where $M$ is the resolution level of the mesh. Copy fields from child meshes back to the root mesh, for the time steps of the latter.

Step 4) Check whether the maximum time step has been reached. If so, terminate the simulation, otherwise return to Step 1).

The next two sections are aimed at explaining these steps in detail.

\section{Mesh TREe AND Field Update Procedure IN AMR-FDTD}

AMR-FDTD applies the well-known field update equations of FDTD for each mesh, yet the interconnection of the different resolution meshes that march in time at different time steps is an issue to be addressed explicitly. In this section, the types of interfaces that occur in an AMR-FDTD domain are presented, along with their treatment in the update process.

\section{A. Categorization of Boundaries of Child Meshes}

The different categories of child mesh boundaries that may practically occur are shown in Fig. 2. In order to facilitate the presentation of the different cases, a 2-D case is discussed (readily extensible to three dimensions). Consider two child meshes $B_{1}$ and $B_{2}$, embedded in a root mesh $A$. Three separate cases of boundaries can be identified as follows.

Case 1) Segment ea: It is defined here as a physical boundary (PB), including absorbing and/or perfect electric conductor boundary conditions.
Case 2) Segment $c d$ : This is a boundary between "sibling" meshes (SB).

Case 3) Segments $a b, b c, d e$ : These are boundaries between child and parent meshes (CPB).

Evidently, it is possible that a boundary may belong to more than one of the aforementioned categories. Then, its classification is based on the following hierarchy:

$$
\mathrm{PB}>\mathrm{SB}>\mathrm{CPB} \text {. }
$$

\section{B. Update of the Mesh Tree}

In the following, the update of a two-level mesh tree (with root and level 1 meshes) is discussed. The steps outlined here can be recursively extended to mesh trees of more levels, by considering, for example, level 2 meshes as roots for level 3 meshes and so on. As before, $\Delta t$ is the time step of the root mesh, while the child level 1 mesh uses a time step $\Delta t / 2$. Let us consider the order of updates, assuming that the time-marching procedure has reached the time $t=n \Delta t$. As a result, it is assumed that the root mesh contains the updated values of the electric field component grid points at $n \Delta t$ and those of the magnetic field component grid points at $(n-1 / 2) \Delta t$. In addition, the child meshes contain the updated electric and magnetic field values at $n \Delta t$ and $(n-1 / 4) \Delta t$, respectively. Then, the following procedure is applied.

1) Backup magnetic field components of the root mesh at $(n-1 / 2) \Delta t$. Obtain their values at $(n+1 / 2) \Delta t$ by applying the FDTD update equations.

2) Backup the electric field components of the root mesh at $n \Delta t$. Obtain their values at $(n+1) \Delta t$ by applying the FDTD update equations.

3) For each child mesh, apply the update equations to obtain the magnetic field values at $(n+1 / 4) \Delta t$.

4) For each child mesh:

a) Update the interior (nonboundary) electric field grid points to obtain their values at $(n+1 / 2) \Delta t$.

b) Update the boundary electric field grid points, at boundaries of the PB, SB, CPB-type, to obtain their values at $(n+1 / 2) \Delta t$. Obviously, these updates are nontrivial, since they invoke grid points of the root mesh, calculated at time steps of the child mesh. Therefore, interpolation needs to be carried out in a way that is analyzed in the next subsection of this paper.

5) For each child mesh, backup the magnetic field components at $(n+1 / 4) \Delta t$ and obtain their values at $(n+$ $3 / 4) \Delta t$ by applying the FDTD update equations.

6) Repeat steps $4 a$ ) and $4 b$ ) to advance the electric field values of the child meshes to $(t+\Delta t)$.

7) For each child mesh:

a) put the spatially interpolated electric field nodal values at $(n+1) \Delta t$ back to the parent mesh, excluding CPB grid points;

b) put the spatially interpolated magnetic field nodal values at $(n+1 / 2) \Delta t$ back to the parent mesh. 


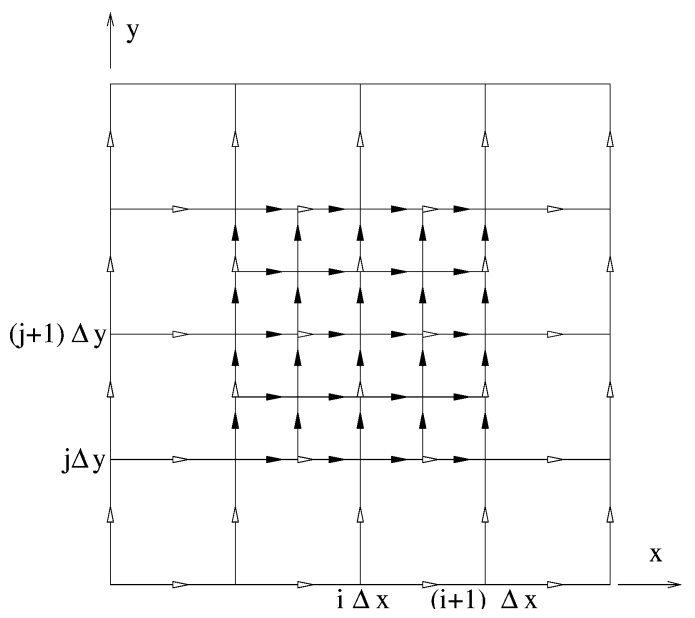

Fig. 3. Sampling points for electric field on a CPB-type boundary. The solid arrows are for the child mesh. The hollow arrows are for the parent mesh.

\section{Field Updates on CPB-Type Boundaries}

The electric field components of a child mesh tangential to its CPB-type boundary [steps $4 a$ ) and $4 b$ )] are obtained from its parent through trilinear interpolation in space and time, as shown in Fig. 3. Since such a boundary is characterized by fixing one spatial variable and letting the other two vary, along with time, trilinear interpolation provides the expression employed in all of these updates. In order to interpolate a function $f(\eta, \xi, \tau)$ in the range $0 \leq \eta, \xi<$ $1,0 \leq \tau<1$, by using values of the function at points $(0,0,0),(0,0,1),(0,1,0),(0,1,1),(1,0,0),(1,0,1),(1,1,0)$, and $(1,1,1)$, the following formula can be applied:

$$
\begin{aligned}
f(\eta, \xi, \tau)= & (1-\eta)(1-\xi)(1-\tau) f(0,0,0) \\
& +\eta(1-\xi)(1-\tau) f(1,0,0) \\
& +\xi(1-\eta)(1-\tau) f(0,1,0) \\
& +\eta \xi(1-\tau) f(1,1,0) \\
& +(1-\eta)(1-\xi) \tau f(0,0,1) \\
& +\eta(1-\xi) \tau f(1,0,1) \\
& +\xi(1-\eta) \tau f(0,1,1)+\xi \eta \tau f(1,1,1) .
\end{aligned}
$$

As an example, consider the $E_{x}$ field on a CPB-type boundary located at $z=0$. The parent mesh has $E_{x}((i+1 / 2) \Delta x$, $j \Delta y, 0, k \Delta t)$, whereas the child mesh needs $E_{x}(x, y, 0, t)$. If $(i-1 / 2) \Delta x \leq x<(i+1 / 2) \Delta x, j \Delta y \leq y<$ $(j+1) \Delta y, k \Delta t \leq t<(k+1) \Delta t$, with $i, j, k$ being integers. Then, $\eta, \xi$ and $\tau$ correspond to the following normalized spatial and temperal variables:

$$
\frac{x}{\Delta x}-i-1 / 2 \quad \frac{y}{\Delta y}-j \quad \frac{t}{\Delta t}-k
$$

which vary within the interval $[0,1)$. Since the child mesh regions $B_{n}$ belong to their root mesh region $A$, the use of (3) allows for the determination of any sampling point of the child mesh from sampling points of its root mesh, enabling the transfer of data which is included in step $4 \mathrm{~b}$ ).

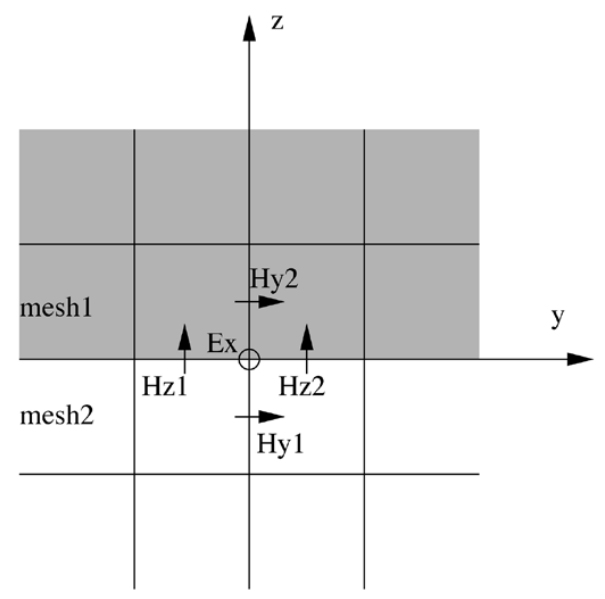

Fig. 4. Interface between two meshes of the same level.

\section{Field Updates on SB-Type Boundaries}

First, note that boundaries between child meshes of different levels of resolution can be treated as the CPB boundaries that were discussed above. Therefore, the treatment of SB-type boundaries can be limited to boundaries between child meshes of the same level. Since, by Yee cell convention, the electric field is sampled at cell edge centers and the magnetic field is sampled at cell face centers, two meshes of the same level share tangential electric field and normal magnetic field components at the interface between them (see, for example, Fig. 4).

However, an inspection of the required grid points that the Yee's algorithm invokes in the update of the field components indicated in Fig. 4 reveals that only the update of the tangential electric field component needs special handling. Referring to Fig. 4, which shows two meshes interfaced at $z=0$, the update of $E_{x}$ in mesh 1 is based on the retrieval of the values of $H_{y_{1}}, H_{y_{2}}, H_{z_{1}}, H_{z_{2}}$. In particular, the index of $H_{y_{1}}$ in mesh 2 should be transparent to mesh 1. For this purpose, the positions of the SB-type boundaries (between child meshes of the same level) are recorded in a table, after each mesh refinement (i.e., at each $N_{\mathrm{AMR}}$ time steps).

One specific difficulty arising in the update of an SB-type boundary comes from $\mathrm{T}$-junction regions, where three sibling meshes share one common edge, and cross-junction regions, where four sibling meshes share one common edge. Ideally, SB-type boundaries should be transparent to incident waves, causing no reflections. However, handling those T-junctions and cross junctions could render the implementation of the algorithm excessively complicated by itself. Therefore, the current implementation treats these junctions as CPB-type boundaries. The numerical results show that any reflections caused by this approach at the junctions are acceptably small.

\section{E. Field Updates on PB-Type Boundaries}

PB-type boundaries include absorbing and/or PEC boundary conditions. These conditions are enforced in both root and child meshes. For the applications that follow, Mur's first-order boundary condition [13] has been used. However, any other type of boundary condition can be readily incorporated. Perfectly matched layer (PML)-type conditions would simply extend the computational domain by the number of the absorber cells. Since 


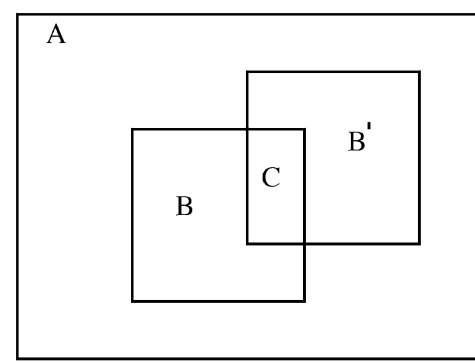

Fig. 5. Transition from old mesh tree to new mesh tree.

PMLs are terminated into PECs, the only type of PB boundaries occurring in a PML-terminated domain are those of PECs.

\section{F. Transition From Old to New Mesh Tree}

Each $N_{\mathrm{AMR}}$ time steps of the root mesh, a new mesh tree is created, representing the adaptive mesh regeneration. Field samples stored in the old mesh tree are transferred to the new mesh tree. This is straightforward for the root mesh, since the new tree has the same root mesh as the old tree. On the other hand, the possibility of an overlap between any child mesh of the new tree and any child mesh of the old tree is checked. If there is such an overlap, the field samples in the overlapping region are transferred from the old to the new child mesh. For example, consider the situation shown in Fig. 5, where $A$ is the root mesh, $B$ is an old child mesh, and $B^{\prime}$ is a new child mesh. $B$ and $B^{\prime}$ overlap in region $C$. Fields of $B$ within $C$ will be copied to $B^{\prime}$ directly, and the rest of $B^{\prime}$ is initialized by interpolating fields of $A$. Note that if $B^{\prime}$ contains boundaries, where conditions, such as Mur's first-order absorbing boundary condition, are applied, and then field values of the current and the last time step are needed. These are maintained and kept available, according to the proposed algorithm of Section III-B. Again, trilinear interpolating operations are employed to initialize the new mesh.

Finally, it is noted that source conditions are always enforced in the root mesh. If a child mesh overlaps with the source region, the overlapping part of the source should also be enforced in the child mesh.

\section{AMR}

Up to this point, the features of AMR-FDTD relevant to the enforcement of a nonuniform grid and the implementation of multiple subgrids within a root mesh have been explained. Standard interpolation operations, which are the common characteristic of any subgridding algorithm, have been proposed. What distinguishes AMR-FDTD from previous subgridding approaches is the AMR, which enables the adaptive movement of the subgrids. This part of the paper explains the core of AMR-FDTD, which is the detection of cells which need further refinement, the dilation of the detected region, to account for wave propagation during the AMR interval, and the clustering of the detected cells, to form child meshes.

\section{A. Detection of Cells That Need Refinement}

Every $N_{\mathrm{AMR}}$-steps, the following calculations are carried out. First, the energy of each $(i, j, k)$ cell of the root mesh is approximated as follows (assume that the computation is made at time step $n$ ):

$$
\begin{aligned}
W_{i, j, k}^{n} & =\frac{1}{2} \int_{V_{i, j, k}}\left\{\epsilon|\bar{E}(\bar{r}, n \Delta t)|^{2}+\mu|\bar{H}(\bar{r}, n \Delta t)|^{2}\right\} d v \\
& \approx \frac{1}{2}\left\{\epsilon_{i, j, k}\left|\bar{E}_{i, j, k}^{n}\right|^{2}+\mu_{i, j, k}\left|\bar{H}_{i, j, k}^{n}\right|^{2}\right\} V_{i, j, k}
\end{aligned}
$$

where $V_{i, j, k}$ is the volume of cell $(i, j, k), W_{i, j, k}^{n}$ is the electromagnetic energy in this cell at time step $n$, and $\bar{E}_{i, j, k}^{n}, \bar{H}_{i, j, k}^{n}$ are vector electric and magnetic field values at the center of the cell at time step $n$, which can be approximated by space/time averaging. Then, the gradient of the energy is numerically approximated by a second-order finite-difference expression, as

$$
\begin{aligned}
\nabla W_{i, j, k}^{n}=\frac{W_{i+1, j, k}^{n}-W_{i-1, j, k}^{n}}{2 \Delta x} & \hat{x}+\frac{W_{i, j+1, k}^{n}-W_{i, j-1, k}^{n}}{2 \Delta y} \hat{y} \\
& +\frac{W_{i, j, k+1}^{n}-W_{i, j, k-1}^{n}}{2 \Delta z} \hat{z} .
\end{aligned}
$$

Defining thresholds $\theta_{g}$ and $\theta_{e}$, a cell $(i, j, k)$ is marked for refinement if both of the following criteria are met:

$$
\begin{aligned}
\left|\nabla W_{i, j, k}^{n}\right| & >\theta_{g} G^{n} \\
W_{i, j, k}^{n} & >\theta_{e} Q^{n}
\end{aligned}
$$

where

$$
\begin{aligned}
G^{n} & =\max _{i, j, k}\left|\nabla W_{i, j, k}^{n}\right| \\
Q^{n} & =\max _{0 \leq m \leq n} W_{a v}^{m} \\
W_{a v}^{m} & =\frac{1}{N_{x} N_{y} N_{z}} \sum_{i, j, k} W_{i, j, k}^{m} .
\end{aligned}
$$

Therefore, the AMR is executed in a cell when both an instantaneous and a calculated over the whole simulated time threshold are exceeded. The first criterion takes into account the appearance of energy gradient peaks, while the second ensures that arbitrary field fluctuations, mainly stemming from numerical errors at the final steps of the simulation, will not create an unnecessary refinement process.

On the other hand, as the AMR-FDTD simulation begins, all of the electric field components assume zero values, except for the ones excited by the source. The detection of cells to be refined at this time-marching stage is difficult, since the energy gradient is too small to surpass the threshold set. To overcome this difficulty, in addition to the cells detected by thresholding, the cells in the source region are also marked for refinement, for a certain period of time. For example, if a Gaussian excitation of the form

$$
\exp \left(-\left(\frac{t-t_{0}}{T_{s}}\right)^{2}\right)
$$

is used (with $t_{0} \sim 3 T_{s}$ ), source region cells are refined up to time $t=2 t_{0}$.

\section{B. Modeling of Wave Propagation}

The application of the previous criteria may result in a number of cells being marked for refinement. However, this refinement process takes place every $N_{\mathrm{AMR}}$ time steps (to avoid loading 


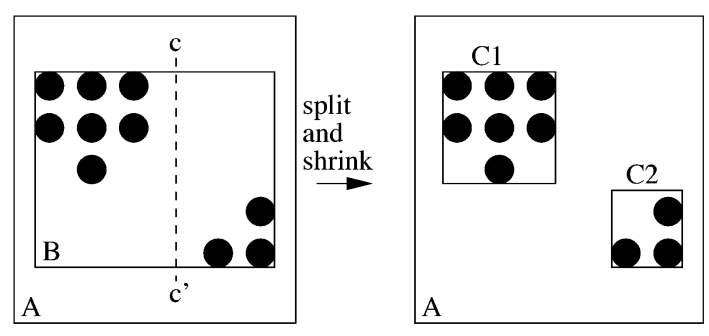

Fig. 6. Splitting and shrinking of boxes (child meshes).

every time step with the mesh refinement operations). Within these time steps, wave propagation within the computational domain can clearly generate the need for a denser mesh in cells neighboring those that are already refined. To capture this field movement, cells within a distance

$$
D=\sigma_{\mathrm{AMR}} N_{\mathrm{AMR}} c \Delta t
$$

from a marked cell are also refined, where $c$ is the speed of light. The factor $\sigma_{\mathrm{AMR}}$ is a predefined, greater-than-one positive real number, which is called the spreading factor. To facilitate computations, the cell-to-cell distances can be defined in the sense of the Manhattan distance $d\left(\bar{r}_{1}, \bar{r}_{2}\right)=\left|x_{1}-x_{2}\right|+\mid y_{1}-$ $y_{2}|+| z_{1}-z_{2} \mid$ instead of the Euclidean distance $d\left(\bar{r}_{1}, \bar{r}_{2}\right)=$ $\left(x_{1}-x_{2}\right)^{2}+\left(y_{1}-y_{2}\right)^{2}+\left(z_{1}-z_{2}\right)^{2}$. Physically, this distance $D$ is the maximum distance that a wave can travel within time equal to $N_{\mathrm{AMR}} \Delta t$, multiplied by the spreading factor. Note that no assumption is being made as to the direction of the wave velocity, which in general is unknown.

The effect of the spreading factor, as well as the thresholds defined in the previous section, will become evident in the numerical results of Section V.

\section{Clustering}

Since the cells that are marked for refinement would generally define irregularly shaped regions, they are first covered by a number of boxes, which are then divided into Yee cells. This procedure is called clustering. To evaluate the quality of clustering, the box coverage efficiency is introduced, which is defined as the ratio of the total volume of the marked cells covered by a box, to the volume of the box.

For the implementation of this clustering procedure, the methodology proposed in [14] is followed. There are three predefined controlling parameters: the threshold for coverage efficiency $\theta_{c}$, the minimum dimension of the box $D_{\min }$, and the maximum number of boxes $N_{\max }$. At the beginning, the bounding box enclosing all the marked cells is found, and its coverage efficiency is calculated. If the coverage efficiency is greater than $\theta_{c}$ or the dimension is less than $D_{\min }$, the algorithm stops; otherwise, the box is split into two boxes across a cut plane. Each of the new boxes is shrunk to just cover the marked cells. Then, the coverage efficiency of each box is calculated and compared with $\theta_{c}$. Again, either box with coverage efficiency less than $\theta_{c}$ and dimension greater than $D_{\min }$ will be split into two. This iterative process continues until the maximum number of boxes is reached.

Fig. 6 illustrates the splitting and shrinking of box B. The black dots represent the marked cells. Box $A$ is the root mesh.
Box $B$ is split across the cut plane $c c^{\prime}$, and the resultant two boxes are shrunk to obtain boxes $C_{1}$ and $C_{2}$. The determination of the position of the cut plane is detailed in [14].

\section{Guidelines for Choosing AMR-FDTD Parameters}

As discussed before, the accuracy and efficiency of AMR-FDTD depends on five parameters: $\theta_{e}, \theta_{g}, \theta_{c}, \sigma_{\mathrm{AMR}}$, and $N_{\mathrm{AMR}}$. While a formal analysis of the dependence of numerical errors of the algorithm on the values of these parameters is beyond the scope of this paper, some empirical guidelines dictating their choice can be provided, based on the authors' experience with the proposed technique.

First, it should be noted that the best achievable accuracy by AMR-FDTD is that of the FDTD scheme applied at a mesh as dense as the finest child mesh in the AMR-FDTD hierarchy of meshes. Therefore, the comments on the accuracy of the technique are meant to be always referred to such an FDTD scheme. Let us also define the AMR coverage as the ratio of mesh-refined regions to the total volume of the computational domain. In general, decreasing the AMR coverage will reduce the execution time of the code but will also reduce its accuracy, since the mesh becomes coarser overall.

Furthermore, $N_{\mathrm{AMR}}$ determines how frequently the AMR operations are performed. Increasing this parameter leads to less AMR-related operations. A subtle side-effect of a large $N_{\mathrm{AMR}}$ is the following: newly generated mesh-refined regions are always extended by a factor proportional to $N_{\mathrm{AMR}}$ [see (10)], to account for wave propagation between successive mesh refinements. Hence, the coverage increases and, thus, so does the execution time. In general, the recommended values of $N_{\mathrm{AMR}}$ are between $10-50$.

The most important source of errors in any static or dynamic mesh refinement scheme is the reflections at a CPB-type boundary. The $\theta_{e}$ parameter directly affects the wave amplitude at such boundaries and as such it is the most important controlling parameter. On the other hand, $\theta_{g}$ ensures that the refined regions enclose large energy gradient variations. In general, $\theta_{e}=0.1-0.5$ and $\theta_{g}=0.01-0.02$.

Finally, a value of $\sigma_{\mathrm{AMR}}=2$ seems to yield satisfactory results in all cases, while the coverage efficiency threshold $\theta_{c}$ is chosen between 0.6-0.8. Note that a large value of the latter enforces the generation of more smaller meshes, thus improving the AMR coverage. At the same time, it generates more CPBand SB-type boundaries, thus increasing the operations related to their management.

These guidelines and the inherent tradeoffs in the choice of the parameters are further illustrated in the numerical results of Section V.

\section{NuMERICAL EXAMPLES}

Two questions remain to be addressed about the AMR-FDTD algorithm. First, what is the effect of the thresholding parameters, defined in the previous sections on the performance and accuracy of the algorithm, and second, whether the overhead that AMR-FDTD accumulates from the application of the mesh refinement can still leave some room for computational savings stemming from a reduced overall number of operations. 


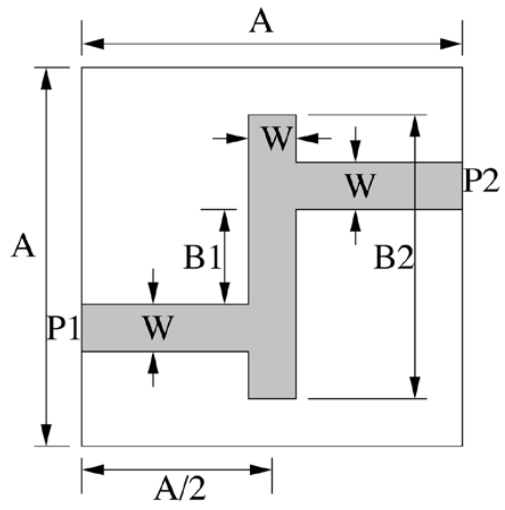

Fig. 7. Microstrip low-pass filter geometry. P1 and P2 denote ports 1 and 2.

Both questions will be addressed through the application of the technique to three microwave circuit geometries, namely, a microstrip low-pass filter, a branch coupler, and a spiral inductor. In all these experiments, a two-level AMR-FDTD is implemented, in order to facilitate the presentation of the effect of its parameters on its accuracy and execution time. Furthermore, an optical waveguide problem, solved with a multiple-level AMR-FDTD, is provided. All simulations were executed on an Intel Xeon 3.06-GHz machine.

\section{A. Microstrip Low-Pass Filter}

The first example is a microstrip low-pass filter, shown in Fig. 7. The dimensions indicated in the figure are $A=40 \mathrm{~mm}$, $B_{1}=2 \mathrm{~mm}, B_{2}=21 \mathrm{~mm}$, and $W=3 \mathrm{~mm}$. The substrate thickness is $0.8 \mathrm{~mm}$ and its dielectric constant is 2.2 . The dimensions of the air box are $40 \mathrm{~mm} \times 40 \mathrm{~mm} \times 4 \mathrm{~mm}$. Two conventional FDTD simulations were performed for comparison: one using a relatively coarse mesh $(40 \times 40 \times 10$ cells $)$ and the second using a dense mesh $(80 \times 80 \times 20$ cells $)$. AMR-FDTD simulations use a $40 \times 40 \times 10$ cell root mesh and different controlling parameters for AMR. Both AMR-FDTD and conventional FDTD use Mur's first-order absorbing boundary condition. A voltage source excitation is imposed at $3 \mathrm{~mm}$ from the edges. In all simulations, a Courant number of 0.7 is used for determining the time step. While AMR-FDTD and the coarse FDTD technique are run for 4096 time steps, the dense FDTD technique uses 8192 steps, since the $\Delta t$ of the latter is equal to one half the $\Delta t$ of the former. The AMR parameters are $\theta_{g}=0.02, \theta_{e}=0.1, \theta_{c}=0.7, N_{\mathrm{AMR}}=10$, and $\sigma_{\mathrm{AMR}}=2$.

To demonstrate the evolution of the child meshes over time, the ratio of the volume of mesh-refined areas (occupied by child meshes) to the total volume (AMR coverage) of the domain and the number of child meshes as a function of the time step are shown in Fig. 8. In addition, Figs. 9-13 show the vertical-toground electric field component $E_{z}$ and child meshes on the plane $z=0.4 \mathrm{~mm}$ at different time steps. The initial child mesh at $t=0$ is given in Fig. 9. As the wave propagates along the feed line, the coverage of refinement increases, until about $t=250 \Delta t$. Between $250 \Delta t-700 \Delta t$, the coverage is relatively large $(40 \%-60 \%)$ due to multiple reflections between the two open ends of the microstrip line. As the fields impinge upon the absorbing boundaries of the structure, the field values in the

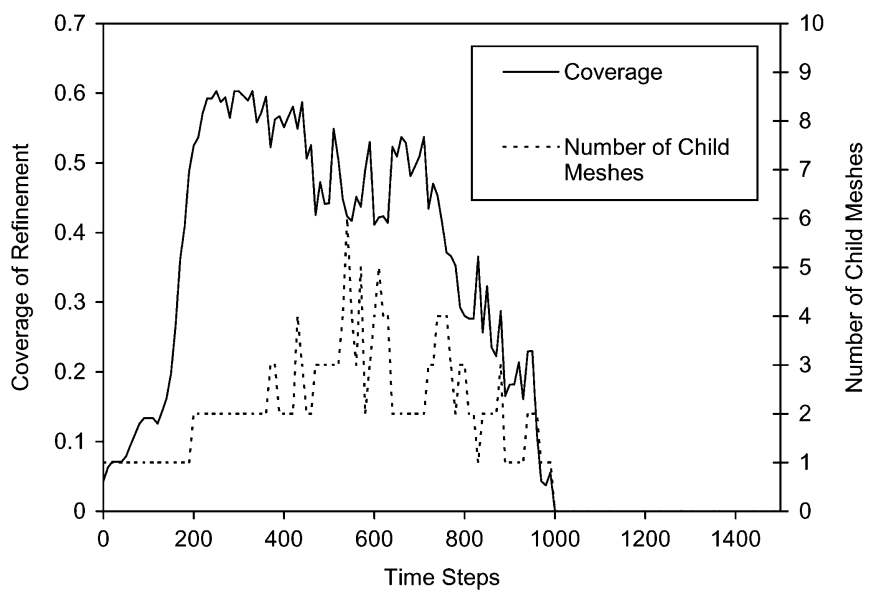

Fig. 8. AMR coverage and number of child meshes at different time steps for the low-pass filter.

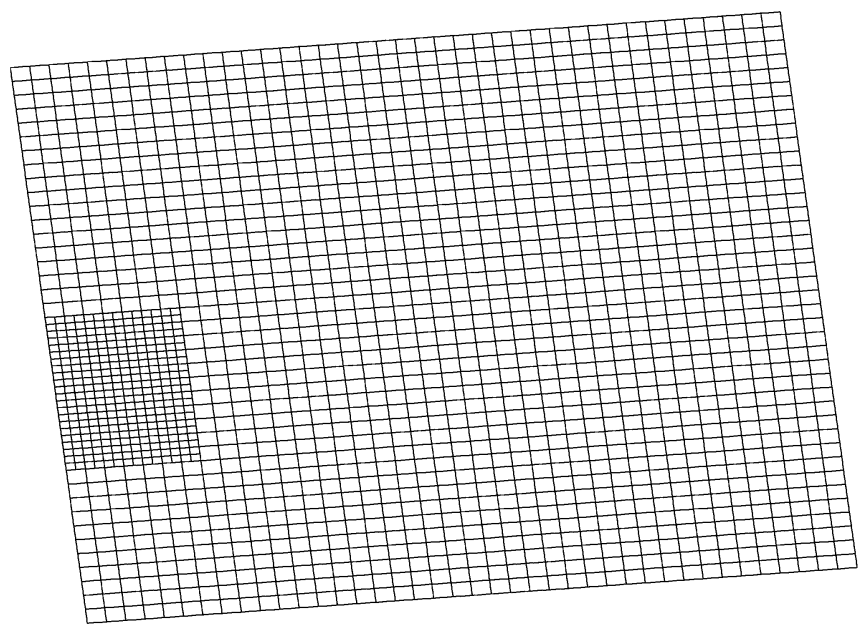

Fig. 9. Vertical electric field magnitude at $z=0.4 \mathrm{~mm}$ and $t=0$. One child mesh; AMR coverage is $4.3 \%$.

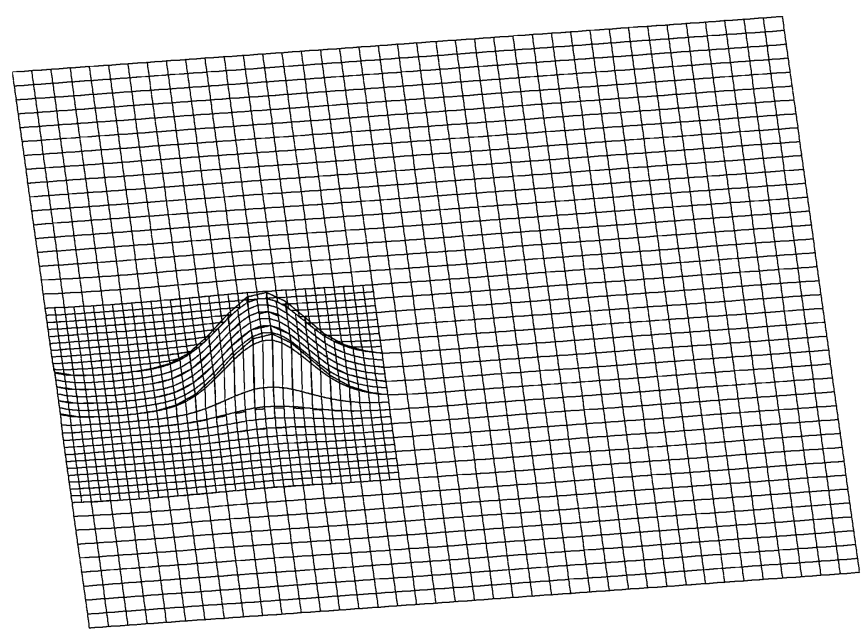

Fig. 10. Vertical electric field magnitude at $z=0.4 \mathrm{~mm}$ and $t=100 \Delta t$. One child mesh; AMR coverage is $13.4 \%$.

working volume of the domain decrease. Consequently, the spatial field variation becomes smoother, which causes the AMR coverage to decrease. In fact, after time $t=1000 \Delta t$, the coarse 


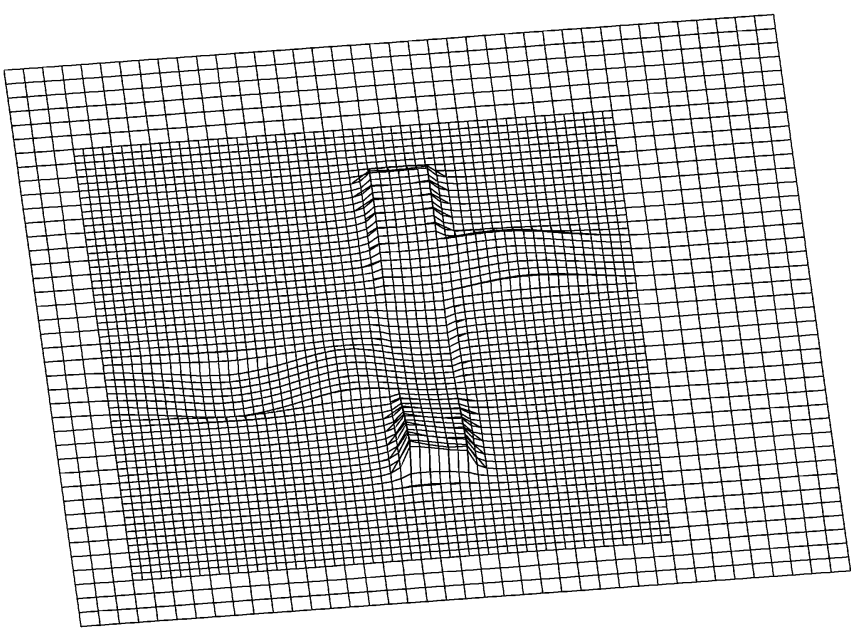

Fig. 11. Vertical electric field magnitude at $z=0.4 \mathrm{~mm}$ and $t=200 \Delta t$. One child mesh; AMR coverage is $52.5 \%$.

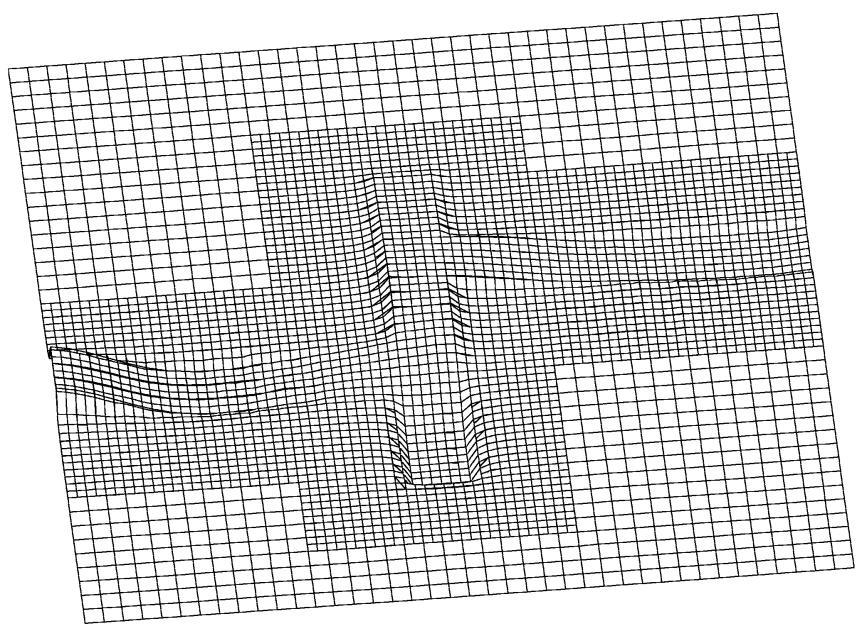

Fig. 12. Vertical electric field magnitude at $z=0.4 \mathrm{~mm}$ and $t=500 \Delta t$. Three child meshes; AMR coverage is $44.2 \%$.

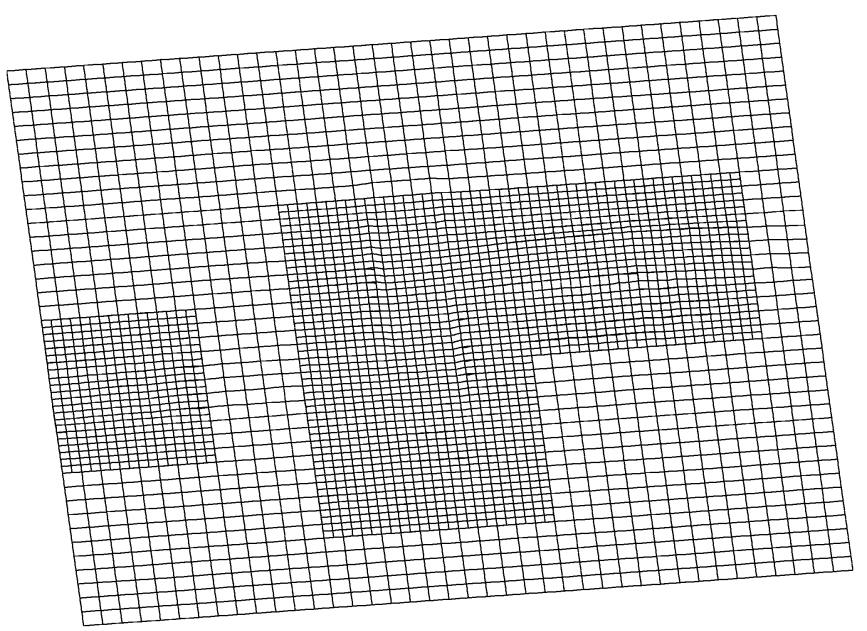

Fig. 13. Vertical electric field magnitude at $z=0.4 \mathrm{~mm}$ and $t=800 \Delta t$. Three child meshes; AMR coverage is $28.0 \%$.

root mesh captures the field solution sufficiently well, so that no child meshes are necessary.
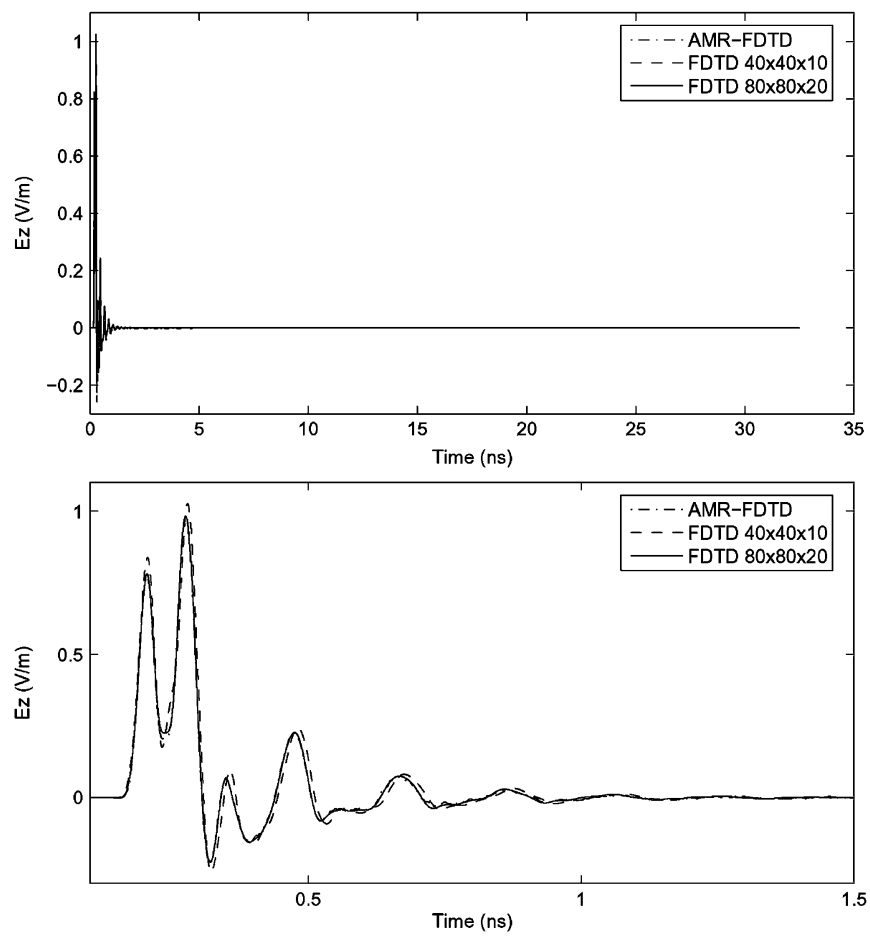

Fig. 14. Vertical electric field magnitude at $z=0.4 \mathrm{~mm}$ and the center of the microstrip line, which is $3 \mathrm{~mm}$ from the right edge.

Fig. 14 compares the vertical electrical field at $z=0.4 \mathrm{~mm}$ and the center of the microstrip line, which is $3 \mathrm{~mm}$ from the right edge up to 40000 time steps ( $32 \mathrm{~ns}$ ). The time series deduced via AMR-FDTD cannot be distinguished from that of the reference FDTD simulation, whereas the result of FDTD using a coarser mesh has a significant difference. The absence of any late-time instability effects is also noted.

The calculated scattering $S$-parameters and their differences are shown in Fig. 15. The plot indicates the excellent approximation provided by AMR-FDTD to the result of FDTD using a dense mesh. This accuracy is quantified in Table I, which employs the following $S$-parameter error metric:

$$
\mathcal{E}_{\mathcal{S}}=\sqrt{\frac{\sum_{k} \sum_{l} \sum_{m}\left|S_{l, m}\left(f_{k}\right)-S_{l, m}^{\mathrm{ref}}\left(f_{k}\right)\right|^{2}}{\sum_{k} \sum_{l} \sum_{m}\left|S_{l, m}^{\mathrm{ref}}\left(f_{k}\right)\right|^{2}}}
$$

where $f_{k}$ is a discrete frequency within the modeled frequency band (up to $30 \mathrm{GHz}$ ), $S_{l, m}$ is the $(l, m)$ element of the scattering matrix of the simulated circuit, as determined by the AMRFDTD or the coarsely meshed FDTD technique, and $S_{l, m}^{\text {ref }}$ is the same element, which is determined by the densely meshed FDTD, which is used as a reference code. From Table I, it is concluded that AMR-FDTD can closely follow the accuracy of the reference FDTD method, while consuming only 5.4\% of its total simulation time. These execution time savings are well above the savings expected from static subgridding algorithms that were previously reported in the literature and indicate the potential of AMR-FDTD.

It should be noted that the ratio between the FDTD simulation times for the fine and the coarse mesh is larger than $16: 1$, which is the ratio between the operations carried out in the two cases. 

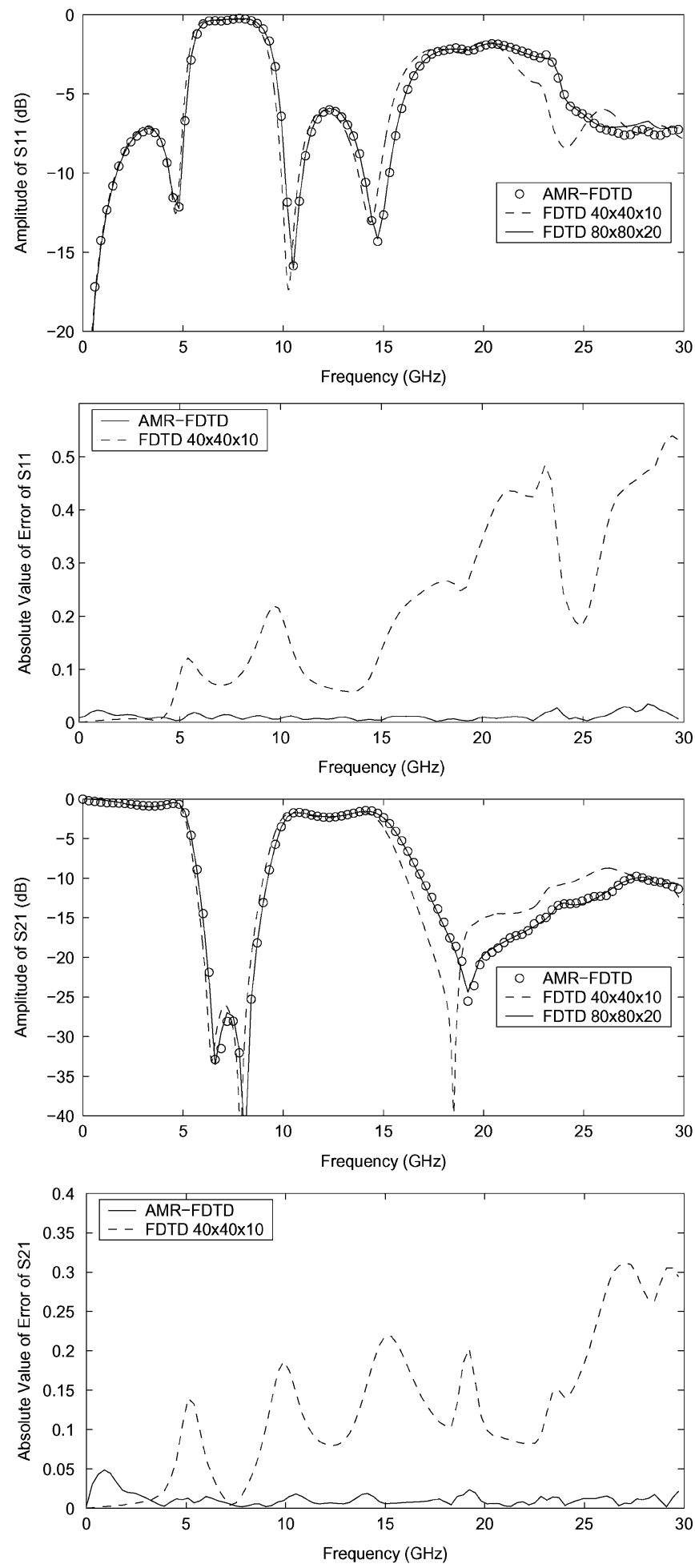

Fig. 15. Comparison of the scattering parameters of the low-pass filter of Fig. 7 obtained by FDTD and AMR-FDTD.

TABLE I

Computation Time and $S$-PARAMETER ERRor Metric $\mathcal{E}_{S}$ FOR THE MICROSTRIP LOW-PASS FILTER

\begin{tabular}{|c|c|c|c|c|}
\hline Method & Mesh & $\begin{array}{l}\text { Time } \\
\text { steps }\end{array}$ & $\begin{array}{l}\text { Total } \\
\text { time }(\mathrm{s})\end{array}$ & $\begin{array}{l}\mathcal{E}_{\text {s }} \\
(\%)\end{array}$ \\
\hline \hline FDTD & $40 \times 40 \times 10$ & 4096 & 75 & 37.3 \\
\hline AMR-FDTD & $40 \times 40 \times 10$ & 4096 & 518 & 2.4 \\
\hline FDTD & $80 \times 80 \times 20$ & 8192 & 9669 & - \\
\hline
\end{tabular}

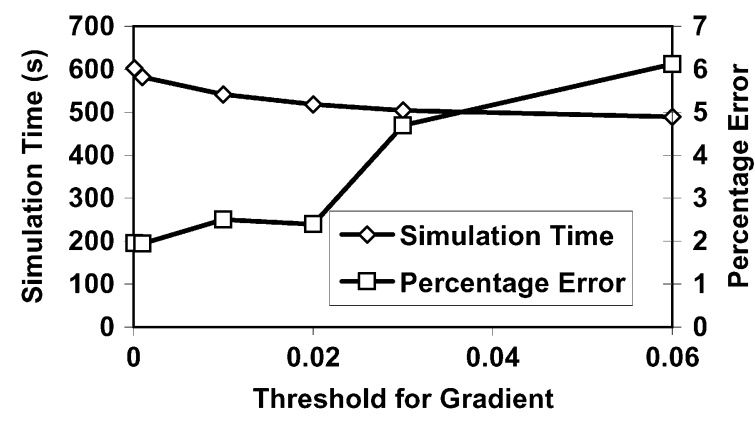

Fig. 16. Effect of threshold for gradient $\theta_{g}$. The rest of the AMR parameters are: $\theta_{e}=0.1, \theta_{c}=0.7, N_{\mathrm{AMR}}=10$, and $\sigma_{\mathrm{AMR}}=2$.

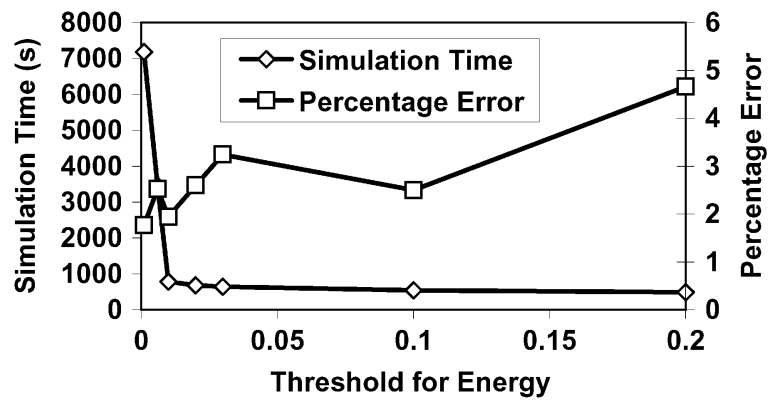

Fig. 17. Effect of threshold for energy $\theta_{e}$. The rest of the AMR parameters are: $\theta_{g}=0.01, \theta_{c}=0.7, N_{\mathrm{AMR}}=10$, and $\sigma_{\mathrm{AMR}}=2$.

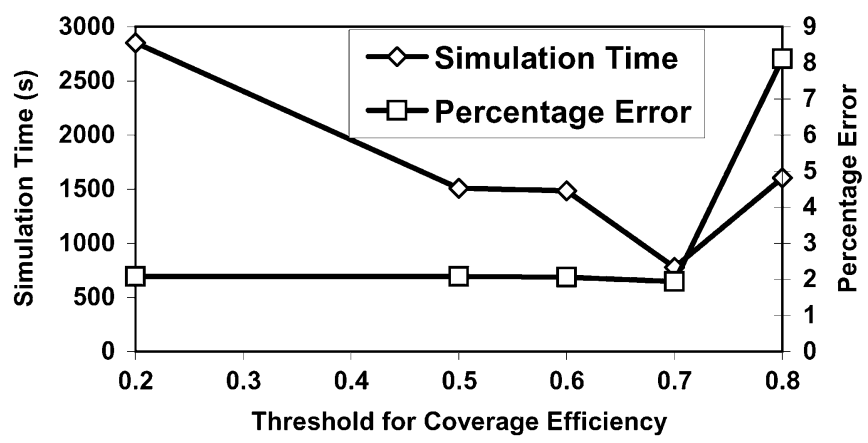

Fig. 18. Effect of threshold for coverage efficiency $\theta_{c}$. The rest of the AMR parameters are: $\theta_{g}=0.01, \theta_{e}=0.01, N_{\mathrm{AMR}}=10$, and $\sigma_{\mathrm{AMR}}=2$.

Our simulation results show that, for small meshes, the simulation time tends to follow the $16: 1$ rule. However, once the mesh size exceeds a certain limit, the simulation time increases faster than $16: 1$. This is due to the increase in the memory access time for large meshes, which further extends their simulation time. A thorough investigation of memory-cache related effects in the execution time of time-discrete methods can be found in [15].

The effect of the AMR-FDTD controlling parameters on the simulation time and error of this technique is studied next and results are shown in Figs. 16-20. As expected, the decrease in $\theta_{g}$ and $\theta_{e}$ results in lower errors and longer simulation times. Essentially, as these two thresholds are lowered, the AMR-FDTD tends to become equivalent in operation and performance to the dense FDTD method. On the contrary, increasing these thresholds can reduce the overall computation time, without sacrificing accuracy, up to some point. It should be noted that the percentage error does not decrease monotonically as $\theta_{g}$ and $\theta_{e}$ decrease. This is because certain child meshes generated by 


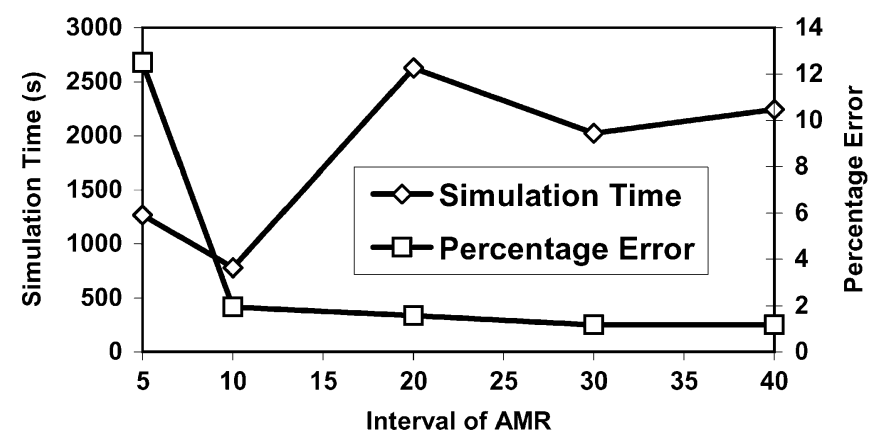

Fig. 19. Effect of interval of AMR $N_{\mathrm{AMR}}$. The rest of the AMR parameters are: $\theta_{g}=0.01, \theta_{e}=0.01, \theta_{c}=0.7$, and $\sigma_{\mathrm{AMR}}=2$.

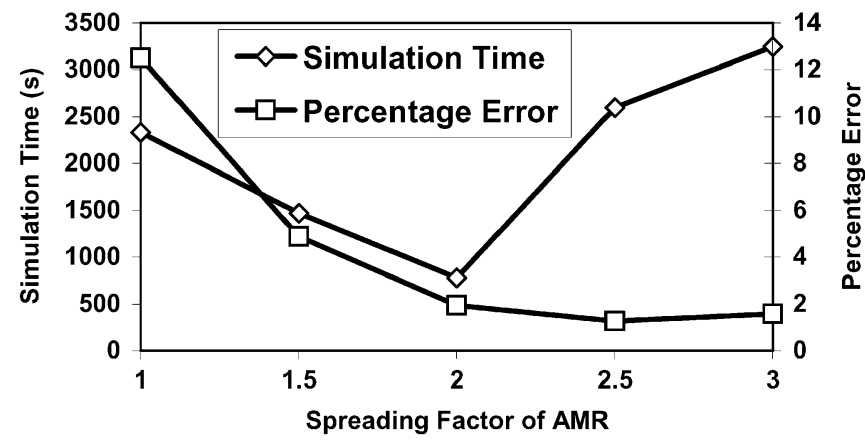

Fig. 20. Effect of spreading factor of AMR $\sigma_{\mathrm{AMR}}$. The rest of the AMR parameters are: $\theta_{g}=0.01, \theta_{e}=0.01, \theta_{c}=0.7$, and $N_{\mathrm{AMR}}=10$.

using smaller $\theta_{g}$ or $\theta_{e}$ may have larger reflections due to the irregularity of the shape of the refined regions. This problem can be alleviated by using higher order interpolation schemes at the mesh boundaries to reduce the reflections [4]-[6]. It is also noted that there is a sudden increase in the simulation time when $\theta_{e}$ is below 0.01 without any associated improvement in accuracy. At the late stage of the simulation, the field components assume some small values due to the reflections at the CPB-and SB-type boundaries. If $\theta_{e}$ is very small, a large number of child meshes can be generated as a result. Subsequently, the simulation time increases, without any improvement in accuracy. An appropriate choice of $\theta_{e}$ can essentially eliminate this problem. Also, when $\theta_{c}$ reaches 0.8 , both the error and the computation time increase. The reason is that the numerical error triggers the automatic generation of multiple spurious child meshes that are clustered independently. As a result, their management by the algorithm adds an overhead that completely eliminates any savings due to the mesh refinement. Tradeoff effects related to the choice of $N_{\mathrm{AMR}}$ and $\sigma_{\mathrm{AMR}}$ are also evident in Figs. 19 and 20. The appropriate values for $N_{\mathrm{AMR}}$ and $\sigma_{\mathrm{AMR}}$, indicated by these plots, have been used in all the aforementioned numerical experiments.

\section{B. Microstrip Branch Coupler}

The microstrip branch coupler geometry of Fig. 21 is analyzed next. The geometric parameters indicated in the figure are as follows: $A=40 \mathrm{~mm}, w_{1}=2 \mathrm{~mm}, w_{2}=3 \mathrm{~mm}$, $B_{1}=7 \mathrm{~mm}, B_{2}=11 \mathrm{~mm}$, and $B_{3}=9 \mathrm{~mm}$. The thickness of the substrate is $0.8 \mathrm{~mm}$, and its dielectric constant is 2.2 . The

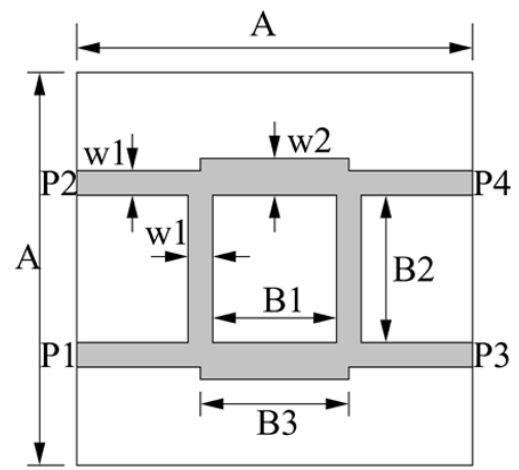

Fig. 21. Microstrip branch coupler geometry. P1, P2, P3, and P4 denote ports $1-4$.
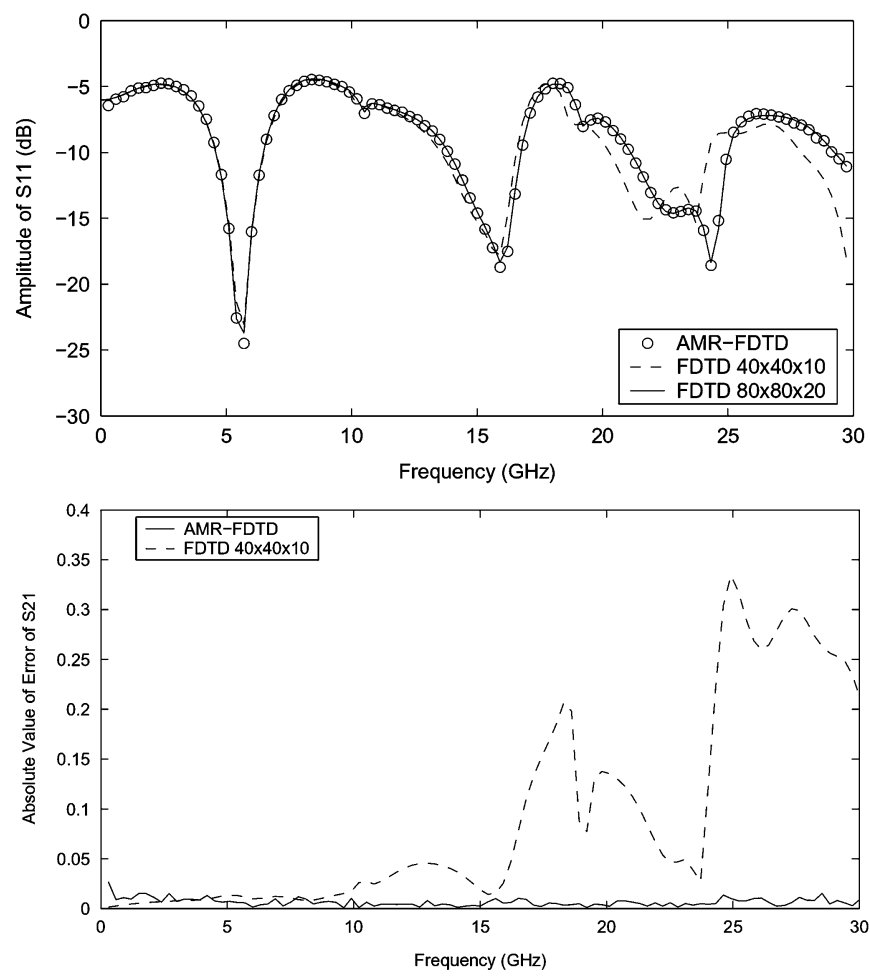

Fig. 22. $S_{11}$ for the microstrip branch coupler geometry of Fig. 21, as determined by FDTD and AMR-FDTD.

dimensions of the computational domain enclosing the structure are $40 \mathrm{~mm} \times 40 \mathrm{~mm} \times 4 \mathrm{~mm}$. The AMR-FDTD method uses a $40 \times 40 \times 10$ mesh and takes 4096 time steps. A reference FDTD simulation of a $80 \times 80 \times 20$ mesh is used for comparison. The AMR control parameters are $\theta_{g}=0.01, \theta_{e}=$ $0.1, \theta_{c}=0.7, N_{\mathrm{AMR}}=10$, and $\sigma_{\mathrm{AMR}}=2$. Figs. $22-25$ show the $S$-parameters and their errors, as determined by the AMR-FDTD, plain FDTD executed on the AMR root mesh, and the reference FDTD simulation. Comparisons regarding computation times and numerical errors of the AMR-FDTD are included in Table II. The data demonstrate AMR-FDTD's ability to deduce the dense FDTD results with a greatly reduced computational effort, which is reflected on an execution time reduction by a factor of 20. Time-domain field waveforms up to 40000 time steps are shown in Fig. 26. Again, the AMR-FDTD and dense-mesh FDTD waveforms coincide, without any sign of late-time instability. 

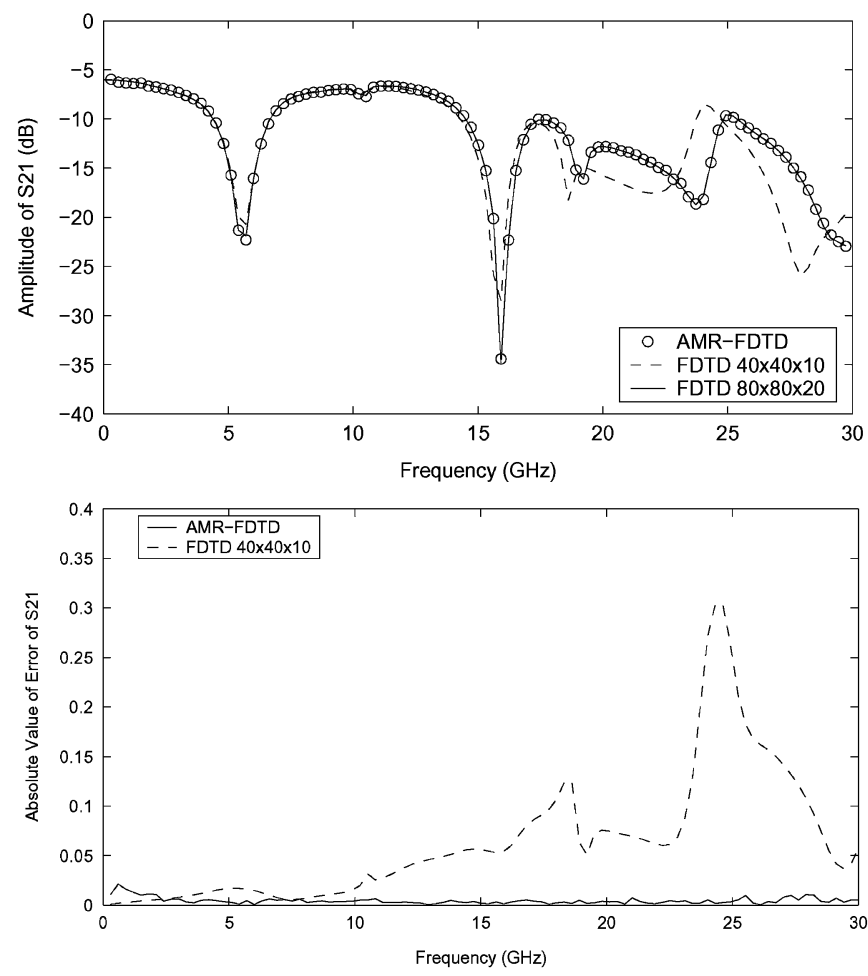

Fig. 23. $S_{21}$ for the microstrip branch coupler geometry of Fig. 21, as determined by FDTD and AMR-FDTD.
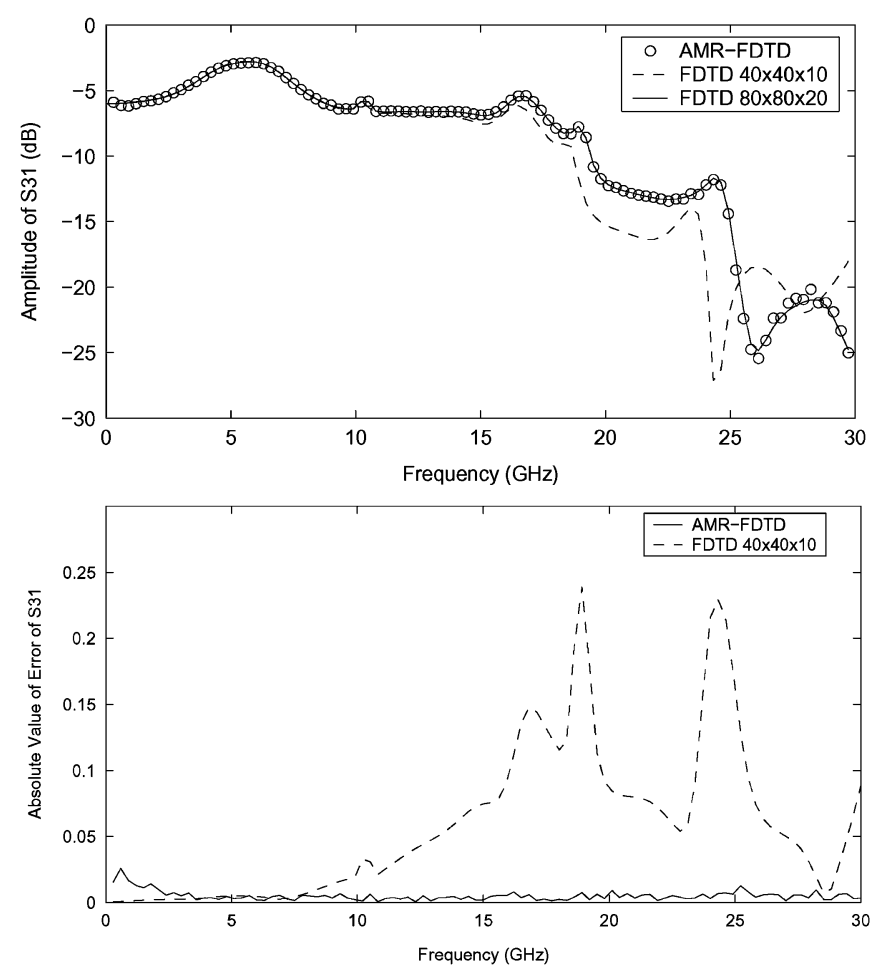

Fig. 24. $S_{31}$ for the microstrip branch coupler geometry of Fig. 21, as determined by FDTD and AMR-FDTD.

\section{Microstrip Spiral Inductor}

As a last example, the geometry of a spiral inductor of Fig. 27 is analyzed. The parameters of this geometry are $A_{1}=60 \mathrm{~mm}$, $A_{2}=40 \mathrm{~mm}, w_{1}=w_{2}=2 \mathrm{~mm}, B_{1}=24 \mathrm{~mm}, B_{2}=$
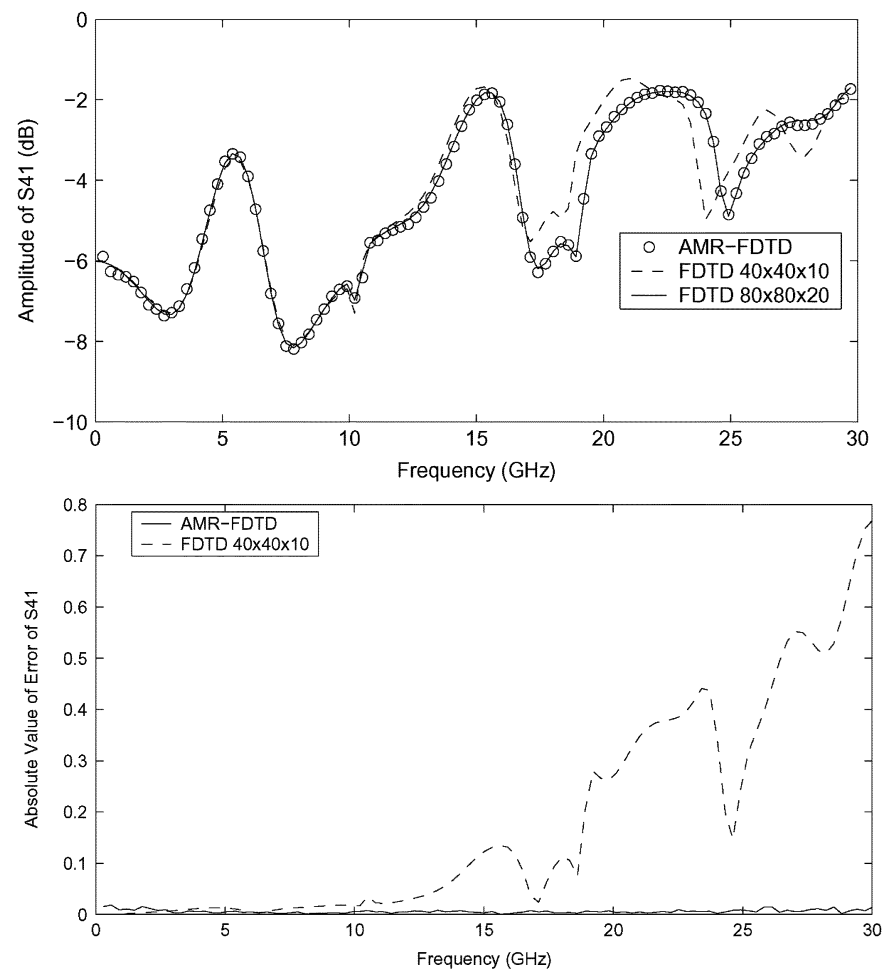

Fig. 25. $S_{41}$ for the microstrip branch coupler geometry of Fig. 21, as determined by FDTD and AMR-FDTD.

TABLE II

Computation Time and $S$-Parameter ERror Metric $\mathcal{E}_{S}$ FOR THE MICROSTRIP BRANCH COUPLER

\begin{tabular}{|c|c|c|c|c|}
\hline Method & Mesh & $\begin{array}{l}\text { Time } \\
\text { steps }\end{array}$ & $\begin{array}{l}\text { Total } \\
\text { time }(\mathrm{s})\end{array}$ & $\begin{array}{l}\mathcal{E}_{s} \\
(\%)\end{array}$ \\
\hline \hline FDTD & $40 \times 40 \times 10$ & 4096 & 262 & 34.6 \\
\hline AMR-FDTD & $40 \times 40 \times 10$ & 4096 & 1021 & 2.43 \\
\hline FDTD & $80 \times 80 \times 20$ & 8192 & 19765 & - \\
\hline
\end{tabular}

$20 \mathrm{~mm}, B_{3}=18 \mathrm{~mm}$, and $B_{4}=4 \mathrm{~mm}$. The thickness of the substrate is $0.8 \mathrm{~mm}$, and its dielectric constant is 2.2 . The dimensions of the computational domain enclosing the structure are $60 \mathrm{~mm} \times 40 \mathrm{~mm} \times 4 \mathrm{~mm}$. The air bridge is $0.8 \mathrm{~mm}$ above the substrate. The AMR-FDTD method uses a $60 \times 40 \times 10$ mesh and 8192 time steps. A reference FDTD simulation of a $120 \times 80 \times 20$ mesh is used for comparison. The AMR control parameters are $\theta_{g}=0.001, \theta_{e}=0.1, \theta_{c}=0.6, N_{\mathrm{AMR}}=$ 50 , and $\sigma_{\mathrm{AMR}}=2$. Fig. 28 shows the $S$-parameters and their errors, as determined by the three methods under comparison, while error and execution time data are shown in Table III. Time-domain results are also shown in Fig. 29.

Compared to the low-pass filter and the branch coupler, the execution time savings achieved by AMR-FDTD over the conventional FDTD are smaller (yet large, at approximately $80 \%$ ), while the associated errors are larger. These effects stem from the highly resonant nature of the spiral inductor, which necessitates the use of a significant number of time steps for the extraction of the $S$-parameters. At late stages of the simulation, relatively small field values are easily contaminated by spurious reflections at the parent-child mesh interfaces. Coupling this method with extrapolation techniques such as Prony's or Matrix Pencil [16] can readily alleviate this problem and maintain the 

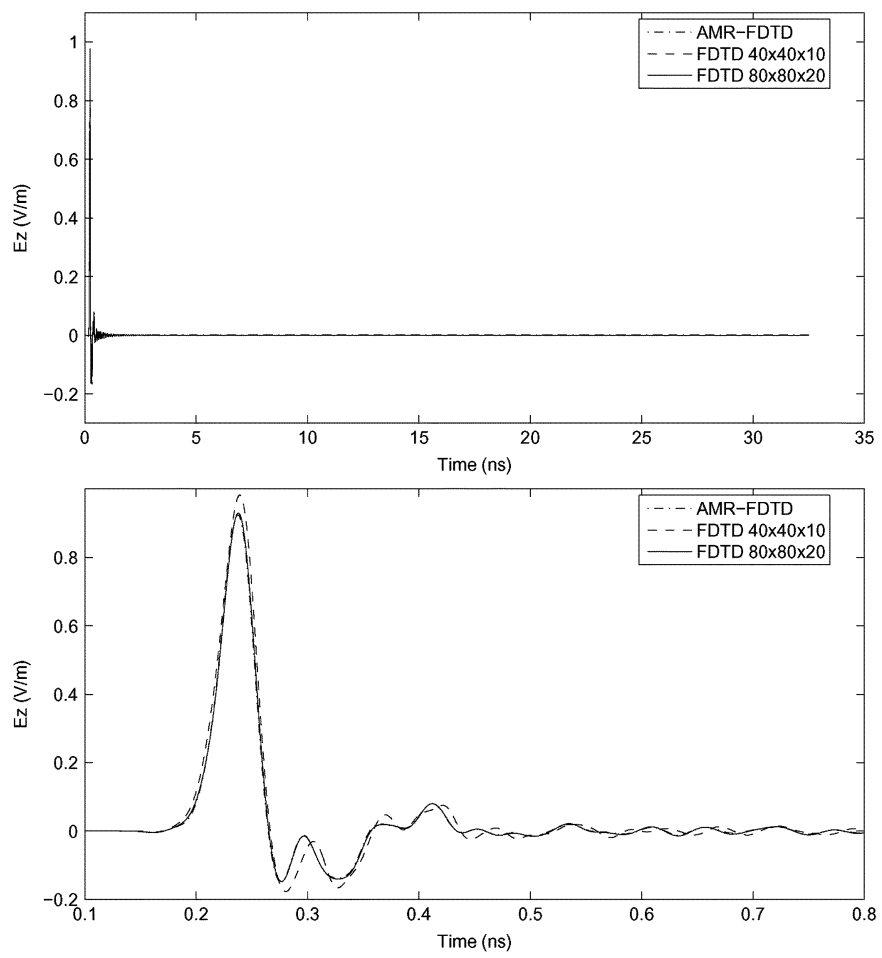

Fig. 26. Vertical electric field magnitude at $z=0.6 \mathrm{~mm}$, and the center of the microstrip line of port 3 is $6 \mathrm{~mm}$ from the edge. The excitation is imposed at $3 \mathrm{~mm}$ from the edge of port 1 .

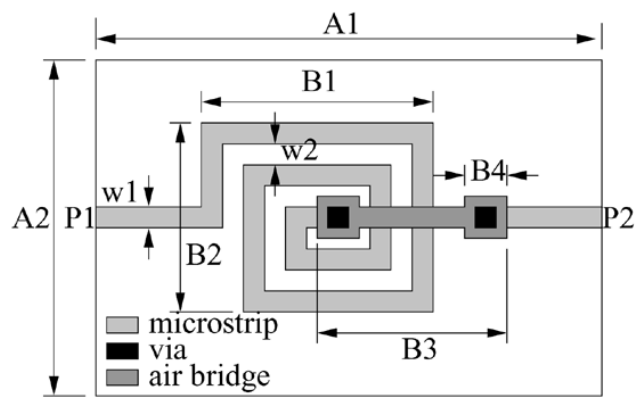

Fig. 27. Spiral inductor geometry. P1 and P2 denote ports 1 and 2 .

numerical advantages of AMR-FDTD. Note that, in this case, the error of the coarse mesh FDTD becomes excessively large as well.

\section{Discussion: Stability and Accuracy of AMR-FDTD Results}

Based on the previous examples, two further comments are in order. First, the time-domain results accompanying the three numerical experiments (see Figs. 14, 26, and 29) demonstrate the absence of late-time instability in the AMR-FDTD. In fact, the convergence of the number of AMR-FDTD child meshes to zero over time implies that only the root mesh is still present at a late stage of the code. Therefore, no spatial or temporal interpolation operations simulation, which are the primary sources of instabilities in adaptive mesh FDTD codes [6], are applied then. This is an additional advantage of using a dynamically adaptive instead of a statically adaptive mesh in time-domain simulations.

Another aspect of the AMR-FDTD accuracy is associated with the reflections at dense/coarse mesh interfaces. A pulse propagating in a statically refined mesh will be reflected off
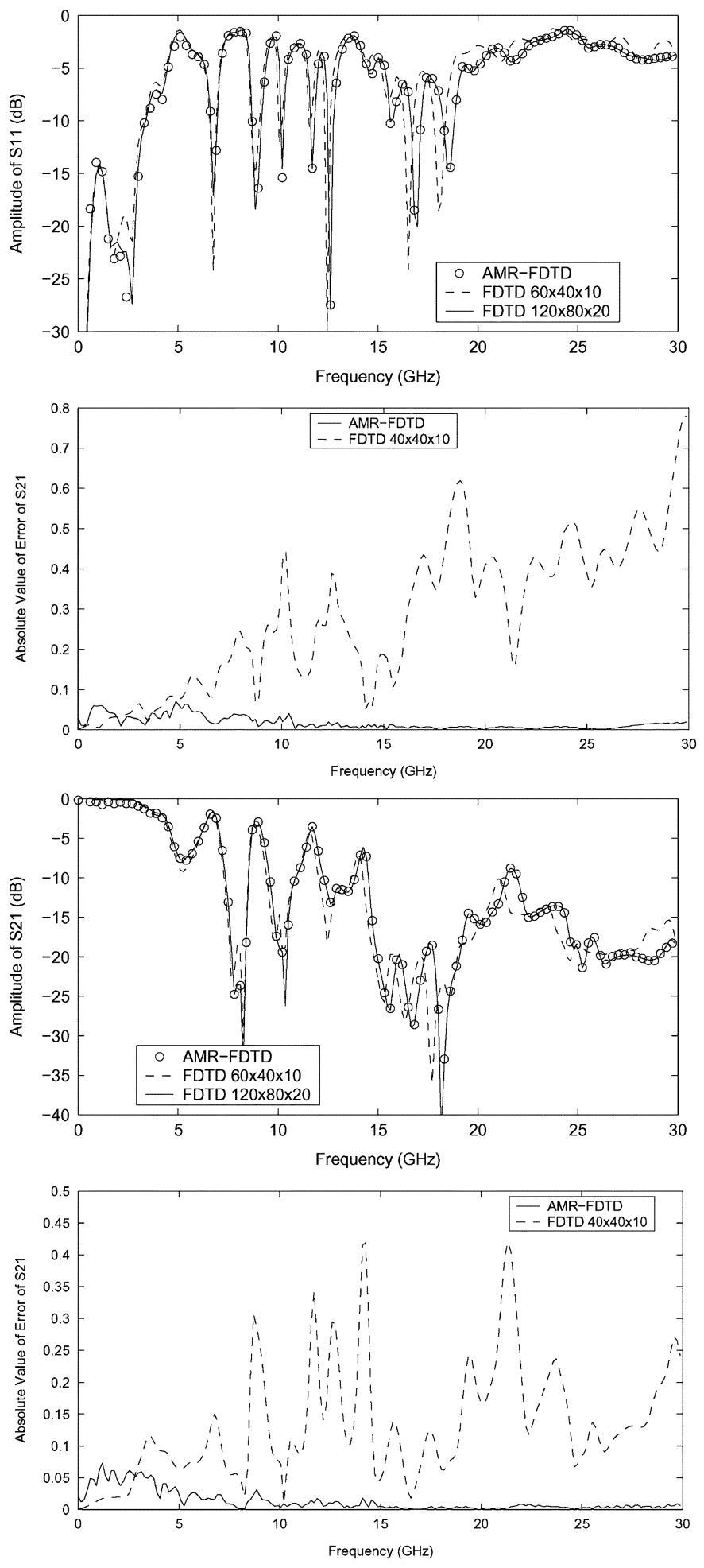

Fig. 28. $S$-parameters for the spiral inductor geometry of Fig. 27, as determined by FDTD and AMR-FDTD.

TABLE III

Computation Time and $S$-Parameter ERror Metric $\mathcal{E}_{S}$ FOR THE MICROSTRIP SPIRAL INDUCTOR

\begin{tabular}{|c|c|c|c|c|}
\hline Method & Mesh & $\begin{array}{l}\text { Time } \\
\text { steps }\end{array}$ & $\begin{array}{l}\text { Total } \\
\text { time }(\mathrm{s})\end{array}$ & $\begin{array}{l}\mathcal{E}_{s} \\
(\%)\end{array}$ \\
\hline \hline FDTD & $60 \times 40 \times 10$ & 8192 & 411 & 52.1 \\
\hline AMR-FDTD & $60 \times 40 \times 10$ & 8192 & 5276 & 3.74 \\
\hline FDTD & $120 \times 80 \times 20$ & 16384 & 29154 & - \\
\hline
\end{tabular}



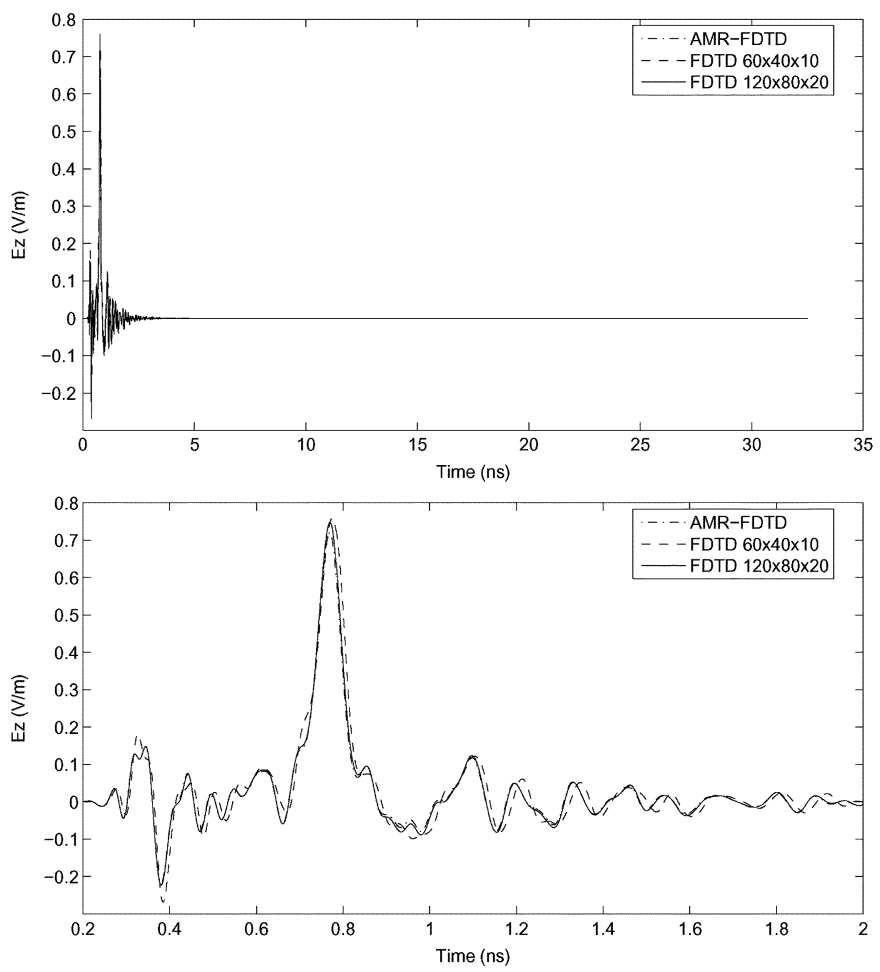

Fig. 29. Vertical electric field magnitude at $z=0.6 \mathrm{~mm}$ and the center of the microstrip line, which is $6 \mathrm{~mm}$ from the right edge. The excitation is imposed at $3 \mathrm{~mm}$ from the left edge of the microstrip line.

such an interface, creating numerical errors. Note that, in AMRFDTD, such a pulse would always be enclosed in a dense mesh, while its retro-reflections might encounter dense/coarse grid interfaces before the AMR algorithm creates new meshes for them. This latter case produces errors in AMR-FDTD, which are evidently smaller than those arising in a static subgrid.

However, the application of interpolations in space and time generates a degradation in the FDTD stability factor. This wellknown effect, also discussed in [6], can be alleviated by using higher order interpolation schemes. For the simple trilinear interpolations employed in this paper, the AMR-FDTD stability limit is observed to be 0.9 of the corresponding FDTD technique in 2-D cases and 0.85 of the corresponding FDTD one in 3-D cases.

Finally, the comparison between coarse/dense FDTD results and AMR-FDTD results in the frequency domain, reveals the standard pattern of the results being in a relatively good agreement with each other up to the middle of the simulated frequency band and diverging afterwards. In these simulations, the coarse grid Yee cell size is about $\lambda_{\min } / 7.5$ is each dimension. Therefore, up to the frequency $f_{\max } / 2$, the so-called coarse mesh uses a sampling rate of at least $\lambda\left(f_{\max } / 2\right) / 15$, which is dense enough to determine the $S$-parameters accurately. From that point on, the effect of the FDTD numerical dispersion becomes more severe, leading to the large errors shown in the figures.

\section{E. Multilevel AMR-FDTD}

To demonstrate the application of the proposed algorithm with multiple resolution levels, a 2-D TE-mode (with an $E_{z}$

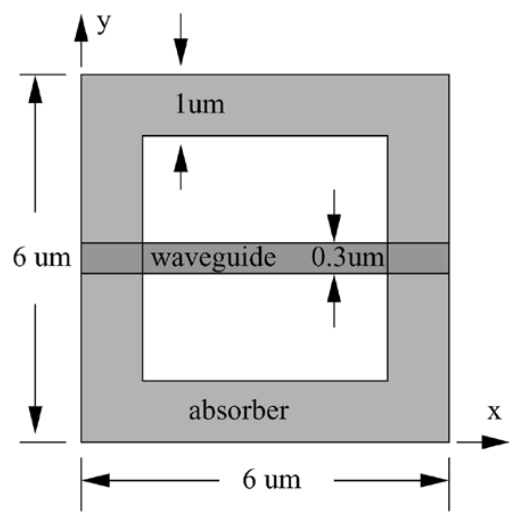

Fig. 30. Optical waveguide geometry.

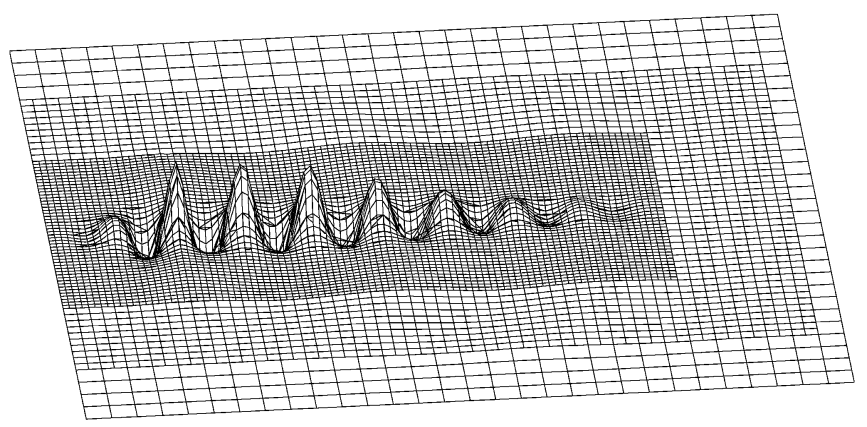

Fig. 31. Vertical electric field magnitude at $t=100 \Delta t$, with one level- 2 child mesh and one level- 3 child mesh.

TABLE IV

Computation Time and Time-Domain ERror Metric $\mathcal{E}_{t}$ FOR THE OPTICAL WAVEGUIDE

\begin{tabular}{|c|c|c|c|c|c|}
\hline Method & Mesh & $\begin{array}{l}\text { Number } \\
\text { of } \\
\text { levels }\end{array}$ & $\begin{array}{l}\text { Time } \\
\text { steps }\end{array}$ & $\begin{array}{l}\text { Total } \\
\text { time } \\
(\mathrm{s})\end{array}$ & $\begin{array}{l}\mathcal{E}_{t} \\
(\%)\end{array}$ \\
\hline \hline FDTD & $120 \times 120$ & - & 2000 & 6.4 & 74.9 \\
\hline FDTD & $240 \times 240$ & - & 4000 & 66.7 & 17.2 \\
\hline FDTD & $480 \times 480$ & - & 8000 & 635 & 3.64 \\
\hline FDTD & $960 \times 960$ & - & 16000 & 13794 & 0.60 \\
\hline FDTD & $1920 \times 1920$ & - & 32000 & 181997 & - \\
\hline AMR-FDTD & $120 \times 120$ & 2 & 2000 & 31.4 & 17.0 \\
\hline AMR-FDTD & $120 \times 120$ & 3 & 2000 & 133 & 3.57 \\
\hline AMR-FDTD & $120 \times 120$ & 4 & 2000 & 641 & 0.56 \\
\hline
\end{tabular}

electric field component only) optical waveguide (shown in Fig. 30) is simulated. Its width is $0.3 \mu \mathrm{m}$, and its dielectric constant is 10.24. The computational domain is $6 \mu \mathrm{m} \times 6 \mu \mathrm{m}$ and a 1- $\mu \mathrm{m}$-thick matched absorber is used to truncate it. Fig. 31 shows a snapshot of the electric field obtained by AMR-FDTD with a maximum number of levels equal to 4 . The excitation is imposed at $1 \mu \mathrm{m}$ from the left edge of the waveguide, and the electric field is recorded at the center of the waveguide and $1.5 \mu \mathrm{m}$ from the right edge. The excitation is a modulated Gaussian pulse of the form

$$
\exp \left(-\left(\frac{y-y_{0}}{W}\right)^{2}-\left(\frac{t-t_{0}}{T_{s}}\right)^{2}\right) \sin (2 \pi f t)
$$

where $T_{s}=0.02 \mathrm{ps}, t_{0}=3 T_{s}, f=200 \mathrm{THz}, W=0.7 \mu \mathrm{m}$, and $y_{0}=3 \mu \mathrm{m}$. Table IV compares the accuracy and computation time of AMR-FDTD using a root mesh of $120 \times 120$ and 2-4 mesh levels, to a reference FDTD simulation using a 


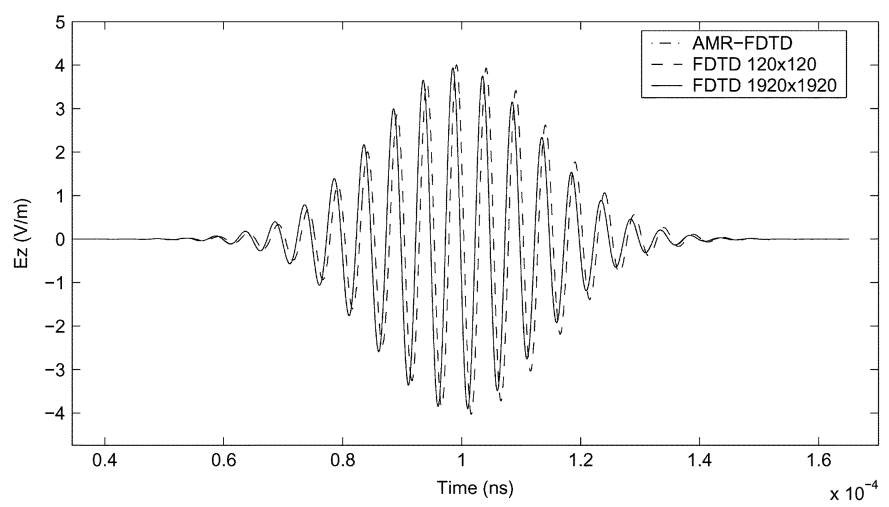

Fig. 32. Vertical electric field magnitude at the center of the optical waveguide, $1.5 \mu \mathrm{m}$ from the right edge.

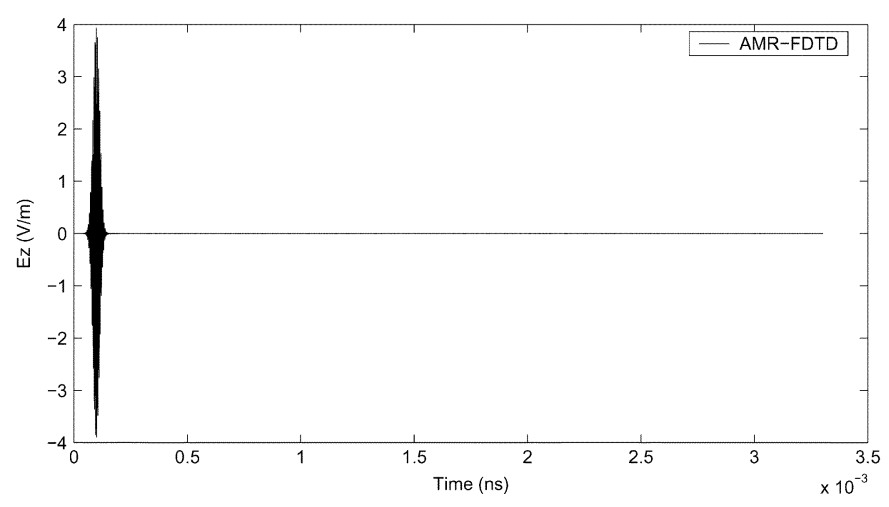

Fig. 33. Vertical electric field magnitude at the center of the optical waveguide, $1.5 \mu \mathrm{m}$ from the right edge,with 40000 time steps.

$1920 \times 1920$ mesh and several coarser FDTD schemes. The accuracy is quantified by employing the following time-domain error metric:

$$
\mathcal{E}_{t}=\sqrt{\frac{\sum_{k}\left|f\left(t_{k}\right)-f^{\mathrm{ref}}\left(t_{k}\right)\right|^{2}}{\sum_{k}\left|f^{\mathrm{ref}}\left(t_{k}\right)\right|^{2}}}
$$

where $t_{k}$ is discrete time within the modeled time range (up to $16 \mathrm{ps}$ ), $f$ is the sampled electric field as determined by AMR-FDTD or the coarse mesh FDTD techniques, and $f^{\text {ref }}$ is the sampled electric field determined by the reference FDTD simulation. All AMR-FDTD simulations use a refinement factor of two for successive levels, and $\theta_{e}=0.1, \theta_{g}=0, \theta_{c}=0.8, N_{\mathrm{AMR}}=10$, and $\sigma_{\mathrm{AMR}}=2$. Fig. 31 shows that AMR-FDTD can reach the accuracy of the reference FDTD method in a greatly reduced computation time. The time-domain simulation results of the AMR-FDTD with four levels and the reference FDTD code are compared in Fig. 32. The AMR-FDTD waveform is not visually discernible from the reference FDTD one. To demonstrate the absence of long-term instability in AMR-FDTD, 40000 time steps of this simulation are also shown in Fig. 33.

\section{CONCLUSION}

In this paper, a dynamic AMR-FDTD technique was presented in detail and applied to realistic microwave circuit and optical waveguide geometries. Essentially, this technique belongs to the class of multiresolution time-domain (MRTD) methods [17], although it does not implicate wavelet basis functions. However, it does implement multiresolution moving grids that enforce a space- and time-adaptive moving mesh in three dimensions, which are regenerated every certain time steps.

The purpose of the applications that were shown, was to demonstrate whether the mesh adaptation overhead of AMRFDTD can still allow for important computational savings. The conclusion is that it does, because the method is optimally suited to the nature of time-domain simulations. The latter are characterized by spatially and temporally localized phenomena that call for a dense mesh not throughout space and time, but only at the certain time and space they happen. Although this paper investigates AMR-FDTD as a microwave-circuit CAD tool, the technique evidently holds a great promise for large-scale optical geometries and wireless problem simulations.

\section{REFERENCES}

[1] K. S. Yee, "Numerical solution of initial boundary value problems involving Maxwell's equations in isotropic media," IEEE Trans. Antennas Propag., vol. AP-14, no. 4, pp. 302-307, Apr. 1966.

[2] D. M. Sheen, S. M. Ali, M. Abouzahra, and J. A. Kong, "Application of the three-dimensional finite-difference time-domain method to the analysis of planar microstrip circuits," IEEE Trans. Antennas Propag., vol. AP-14, no. 7, pp. 302-307, Jul. 1966.

[3] I. S. Kim and W. J. R. Hoefer, "A local mesh refinement algorithm for the time domain-finite difference method using Maxwell's curl equations," IEEE Trans. Microw. Theory Tech., vol. 38, no. 6, pp. 812-815, Jun. 1990.

[4] S. S. Zivanovic, K. S. Yee, and K. K. Mei, "A subgridding method for the time-domain finite-difference method to solve Maxwell's equations," IEEE Trans. Microw. Theory Tech., vol. 39, no. 3, pp. 471-479, Mar. 1991.

[5] M. W. Chevalier, R. J. Luebbers, and V. P. Cable, "FDTD local grid with material traverse," IEEE Trans. Antennas Propag., vol. 45, no. 3, pp. 411-421, Mar. 1997.

[6] M. Okoniewski, E. Okoniewska, and M. A. Stuchly, "Three-dimensional subgridding algorithm for FDTD," IEEE Trans. Antennas Propag., vol. 45, no. 3, pp. 422-429, Mar. 1997.

[7] R. Luebbers, J. Schuster, and K. Wu, "Application of moving window FDTD to prediction of path loss over irregular terrain," in Proc. IEEE AP-S Int. Symp., vol. 2, Jun. 2003, pp. 610-613.

[8] M. J. Berger and J. R. Oliger, "Adaptive mesh refinement for hyperbolic partial differential equations," J. Comput. Phys., vol. 53, pp. 484-512, 1984.

[9] E. Bacry, S. Mallat, and G. Papanicolaou, "A wavelet based space-time adaptive numerical method for partial differential equations," Math. Modeling Numer. Anal., vol. 26, no. 7m, p. 793, 1992.

[10] C. D. Sarris and L. P. B. Katehi, "Coupling front tracking and wavelet techniques for fast time domain simulations," in IEEE MTT-S Int. Microw. Symp. Dig., vol. 2, pp. 747-750.

[11] Y. Liu and C. D. Sarris, "AMR-FDTD: A dynamically adaptive mesh refinement scheme for the finite-difference time-domain technique," presented at the IEEE AP-S Int. Symp., 2005.

[12] - "Application of AMR-FDTD to microwave integrated circuits," presented at the Eur. Microw. Conf., 2005.

[13] G. Mur, "Absorbing boundary conditions for the finite difference approximation of the time domain electromagnetic field equations," IEEE Trans. Electromagn. Compat., vol. EMC-23, no. 6, pp. 377-382, Nov. 1981.

[14] M. Berger and I. Rigoutsos, "An algorithm for point clustering and grid generation,” IEEE Trans. Syst., Man, Cybern., vol. 21, no. 5, pp. 1278-1286, Sep./Oct. 1991.

[15] T. Mangold, J. Rebel, W. J. R. Hoefer, P. P. M. So, and P. Russer, "What determines the speed of time-discrete algorithms," in Proc. 16th Annu. Rev. Progress Appl. Computat. Electromagn., vol. 2, Monterey, CA, Mar. 2000, pp. 594-601. 
[16] Y. Hua and T. K. Sarkar, "Matrix pencil method for estimating parameters of exponentially damped/undamped sinuoids in noise," IEEE Trans. Acoust., Speech Signal Process., vol. 38, no. 5, pp. 814-824, May 1990.

[17] M. Krumpholz and L. P. B. Katehi, "MRTD: New time domain schemes based on multiresolution analysis," IEEE Trans. Microw. Theory Tech., vol. 44, no. 4, pp. 555-561, Apr. 1996.

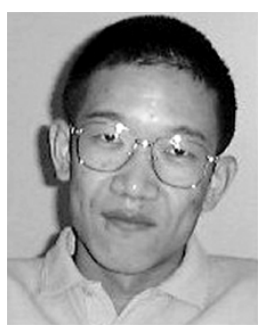

Yaxun Liu (S'00-M'05) received the B.Eng. degree in information and control engineering from Xi'an Jiaotong University, Xi'an, China, in 1994, the M.Eng. degree in electrical engineering from National University of Singapore, Singapore, in 2001, and the Ph.D. degree in electrical engineering from the University of Waterloo, Waterloo, ON, Canada, in 2005 .

He is currently a Post-Doctoral Fellow with the Edward S. Rogers Sr. Department of Electrical and Computer Engineering, University of Toronto, Toronto, ON, Canada. His research interests include computational electromagnetics, microwave and optical integrated circuits, signal integrity, dielectric resonators, microstrip antennas, and dispersive and multilayered media.

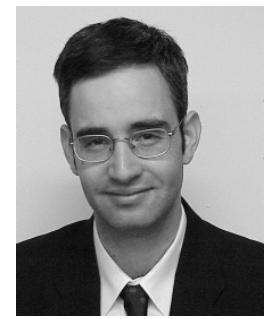

Costas D. Sarris (M'03) received the Diploma degree (with distinction) in electrical and computer engineering from the National Technical University of Athens (NTUA), Athens, Greece in 1997, and the M.Sc. degree in electrical engineering, M.Sc. degree in applied mathematics, and Ph.D. degree in electrical engineering from The University of Michigan at Ann Arbor, in 1998, 2002, and 2002, respectively.

In November 2002, he joined the Edward S. Rogers Sr. Department of Electrical and Computer Engineering (ECE), University of Toronto, Toronto, ON, Canada, where he is currently an Assistant Professor. His research interests are in the area of computational electromagnetics, with emphasis in high-order mesh-adaptive techniques. He is currently involved with basic research in novel numerical techniques, as well as applications of time-domain analysis to wireless channel modeling, wave-propagation in meta-materials and electromagnetic compatibility/electromagnetic interference (EMI/EMC) problems.

Prof. Sarris has been the recipient of a number of scholarship distinctions, including Hellenic Fellowship Foundation (1993-1997) and Technical Chamber of Greece (1994-1997) Awards for academic excellence and an NTUA 1997 Class Bronze Medal. He was also the recipient of a Student Paper Award presented at the 2001 International Microwave Symposium for his research on a hybrid FDTD/MRTD numerical scheme and a Canada Foundation for Innovation New Opportunities Fund Award in 2004. 